\title{
Assessing The Sustainable Safety Practices Based On Human Behavior Factors: An Application to Chinese Petrochemical Industry
}

\author{
Junqiao Zhang
}

University of Science and Technology Liaoning

Qiang Qu

University of Science and Technology Liaoning

Xuebo Chen ( $\square$ xuebochen@126.com )

University of Science and Technology Liaoning

\section{Research Article}

Keywords: Petrochemical Industry, Sustainable Safety Development, BBS, FAHP, Human Factors, FCE

Posted Date: November 15th, 2021

DOI: https://doi.org/10.21203/rs.3.rs-945108/v1

License: (c) (i) This work is licensed under a Creative Commons Attribution 4.0 International License.

Read Full License

Version of Record: A version of this preprint was published at Environmental Science and Pollution Research on February 8th, 2022. See the published version at https://doi.org/10.1007/s11356-02218909-5. 

petrochemical industry

\author{
Junqiao Zhang ${ }^{1}$, Qiang $\mathrm{Qu}^{1}$ and Xue-bo Chen ${ }^{1 *}$ \\ ${ }^{1}$ School of Electronics and Information Engineering, University of Science and Technology \\ Liaoning, Anshan, China \\ xuebochen@126. com
}

\begin{abstract}
.
Many catastrophic accidents and irreversible environmental pollution have occurred in the petrochemical industry, most of which are caused by human factor errors. Therefore, sustainable safety development is one of the main tasks of petrochemical industry. Behavior-based safety (BBS) management is an effective means to improve human factor errors and reduce accidents. Previous BBS literature studies mainly focused on single method, while rarely combined with other quantitative technical methods. This study proposes an extended BBS approach that combines the application of fuzzy analytic hierarchy process (FAHP) and fuzzy comprehensive evaluation (FCE) to assess sustainable safety performance of a petrochemical industry. By using the FAHP for design and prioritizing the behavior factors to develop a BBS program and provide a guidance for the safety managers and employees on site. The FCE method is applied to evaluate the sustainable safety performance and this method is a new application of BBS management. To verify the validity of the proposed framework, the FCE results before and after BBS management are compared. The results show that the approach has a positive effect on human factor errors, accident reduction and sustainable safety development. This paper supplements the knowledge system of BBS program in terms of method and carry out two respects and provides leaders with guidance on sustainable safety development.
\end{abstract}

Keywords: Petrochemical Industry, Sustainable Safety Development, BBS, FAHP, Human Factors, FCE. 


\section{Introduction}

38 Petrochemical industry contains a large number of flammables, corrosive, toxic and explosive materials (Rollinson, 2018). A small accident may cause irreversible environmental pollution, casualties and societal losses. Review of accident data in recent years shows that fires, combustible dust explosion, petrochemical leakages, factory explosions and suffocations have occurred in the petrochemical industry (wang et al. 2020). Furthermore, unsafe production in the petrochemical industry may lead to environmental pollution, energy waste, climate change and oil price change, which are regarded as a great threat to sustainable safety development (Sharma et al. 2017). Therefore, it is vital to protect the environment and reduce accidents for move towards sustainable safety development. In order to achieve these sustainable safety development goals, three aspects need to be considered: environment, society and economy (Santoyo-Castelazo and Azapagic, 2014). This is gradually recognized by decision makers and managers, and is reflected in some accident studies. However, the importance of human factors has been fully established in the causes of occupational injuries and accidents (Baysari et al. 2008; Kelly and Efthymiou 2019; Karthick et al. 2020).

In the petrochemical industry, the consequences of major accidents may be disastrous, the environment may need to be remedied quickly, and the economic impact can be significant for the country and enterprises. (Harsini et al. 2020). The employee's unsafe behaviors in their daily works can have a direct and indirect impact on workplace safety and fatalities (Dodoo and Samarraie 2021). Among the direct causes of accident, Heinrich believes that 88 percent are unsafe behavior of persons, 10 percent are unsafe situations, and 2 percent are unpreventable (Heinrich 1931). Reason claims that human behaviors are a root cause of accidents; these behaviors are sometimes deliberate and other times, inadvertent (Reason 2000). Garlapati et al. support this stated that unsafe acts of persons cause most of the accidents and occupational injuries in the oil and gas sectors (Garlapati et al. 2013). It therefore follows that, if unsafe behaviors can be modified, accidents and occupational injuries will be reduced as well. Although in subsequent causation models, the cause of the accident extends to supervisors and senior managers such as the Swiss cheese model (Reason et al. 2006) and Loss Causation Model (Chua and Goh 2004), understanding and improving unsafe behavior is still one of the significant research subjects in sustainable safety development.

The two effective management methods are found to be a safety culture-based approaches and behavior-based safety. Thus, one effective approach used to improve unsafe acts of persons is behavior-based safety (BBS) (Geller 2005; Navidian et al. 2015). BBS has been successfully applied in various area in North America, Asia and Europe for over the past years such as mining (Hagge et al. 2017), the construction industry (Lee et al. 2019), robots (Scianca et al. 2021), the E-waste collection (Batoo et al. 2021) and the vehicle industry (Wang et al. 2018). The above researches demonstrate the effectiveness of BBS management on improving unsafe behaviors and sustainable safety performance. There is no agreed concept of Behavior-Based Safety (BBS), but it is usually considered as an approach aiming at safety intervening and modifying unsafe acts of persons (Geller 1999; Geller 2002). The BBS approach is established on correc- 
80 tive feedback and positive observation rather than punitive measures, which the workers get after being observed immediately by colleagues or managers. The approach may include corrective feedback for unsafety behavior and proposals on how to promote safety acts (Brosschot et al. 2018). The combination of the safety observation, goal setting and feedback process together with management support is aimed to identification critical unsafe behavior, promote safety climate and reduce occupational injuries and accidents. Furthermore, safety behaviors have become play an important mechanism in preventing or reducing serious consequence such as occupational fatalities and casualty (Li et al. 2020). Safety behavior is defined as the act of improving safety and health for employees, the community, and the work environment (McSween and Moran 2017). As a result, more and more research has focusing on analyzing and identifying safety behavior as a tool for reducing industry accidents. In particular, it is necessary to strengthen the empirical research on factors affecting employees' unsafe behaviors. This means that more attention should be paid to the analysis and understand of human factors or behaviors (Mirzaei et al. 2020), such as stress, training, experience, etc. (Sauer et al. 2016). Therefore, an effective way to improve people's unsafe behavior is BBS.

These researches provide support for the concentrate on participation in a BBS management. Furthermore, BBS management provides a procedural approach to construct sustainable safety performance gains and process control. BBS theory claim that as employee's safety awareness and behavior habits are not inherent, therefore, they can be improved with education and training (Feng et al. 2020), and the successful implementations of the BBS method appear significant. Although BBS has many successful cases, others point out that there is still room for improvement (Alkaissy et al. 2020). For instance, in the petrochemical and petroleum industry, the quantitative assessment and analysis of the behavior of employees is still scarcely considered. In fact, the improvement of human safety depends on the reduction of the risks for the employees when carry out routine work considering safety behavior (Rodriguez et al. 2017; Dasgupta et al. 2020). In order to reduce the occasional of industry accidents, the study focused on the behavioral and assessment side of the employees, design and implement BBS program, which starts from the understanding and identification of potential unsafe behavior to assess the employees' safety performance (Norton et al. 2017). Therefore, to preferably solve the accidents and occupational injuries, the combination of a fuzzy analytic hierarchy process (FAHP), a BBS and fuzzy comprehensive evaluation (FCE) should be proposed. There are several reasons that we apply the combined method.

Analytic hierarchy process (AHP) is a decision-making method to analyze and solve complex problems based on mathematics and psychology (Nguyen et al. 2015). However, the determinants of sustainable safety performance evaluation contain intangible side of a precise data. It becomes difficult for human to immediately describe and evaluate sustainable safety performance. Since people and preference judgments are usually vague and uncertain, they cannot assess their preference with an accurate numerical value (Pramanik et al. 2020). Additionally, the evaluating factors involve complex and vague internal relationships, leading to a fuzzy evaluation result (Mohsenzadeh et al. 
124 2019). As a result of, it is necessary to utilize a hybrid method to evaluate the sustainable safety performance involving linguistic variables (Ma et al. 2021). Fuzzy set is an effective and appropriate tool to tackle uncertain problems. While, AHP, developed by Saaty, is a reasonable decision-making approach (Saaty 1980). As an extension of AHP, FAHP can solve the problem of fuzzy multi-criteria decision-making and it has been widely applied in various fields, such as project prioritization and selection (Shaygan and Testik 2019; Wang et al. 2019), capital investment (Khashei and Sharifan 2020; Chien et al. 2021), Brand Preference (Hsu et al. 2021), three-dimensional printers (Chen and $\mathrm{Wu} 2021$ ), sustainable production and consumption (Shete et al. 2020; Goyal et al. 2021), manufacturing service (Gul et al. 2018; Hu et al. 2021), oil industry (Iqbal et al. 2021) and city construction (Lyu et al. 2020). So, the FAHP is appropriate for calculating the factors weights in the assessment model. To improve employees' sustainable safety performance, this paper develops a fuzzy management behavior-based assessment model by using FAHP, BBS and FCE (Chang 1992; Chang 1996).

The rest of this paper is organized as follow. In Section 2 and 3, we describe the related definition of FAHP and FCE, respectively. In Section 4, taking a petrochemical plant as an example, the combined application of the FAHP, FCE and BBS management. The main discussions are drawn in Section 5. The conclusions and limitations of the study are given in Sections 6 and 7, respectively.

\section{$2 \quad$ Fuzzy analytic hierarchy process}

144 In this section, we introduce some definition and operational laws correlated to trian145 gular fuzzy number (TFN).

\section{$146 \quad 2.1 \quad$ Triangular fuzzy numbers}

A fuzzy number $A$ on $R$ to be TFN if its membership function $\mu_{\tilde{A}}(x): R \rightarrow[0,1]$ is equal to following Eq. (1) (Chang 1996)

$$
\mu_{\text {Ro }}(x)= \begin{cases}\frac{x-l}{m-l} & \text { for } l \leq x \leq m \\ \frac{u-x}{u-m} & \text { for } m \leq x \leq u \\ 0 & \text { otherwise }\end{cases}
$$

Where $l \leq m \leq u, l$ and $u$ can be expressed the lower and upper values of the respec- 


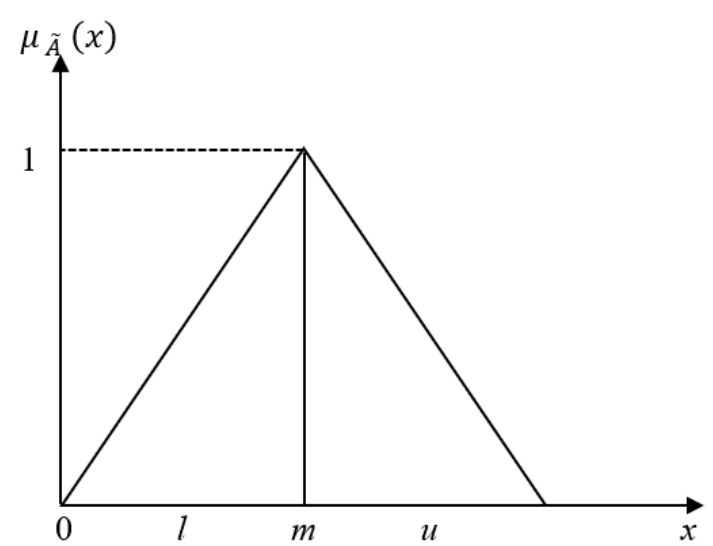

Fig. 1. A triangular fuzzy number, $\tilde{A}=(l, m, u)$.

\subsection{The operational laws of TFN}

Let $\tilde{A}=\left(l_{1}, m_{1}, u_{1}\right)$ and $\tilde{B}=\left(l_{2}, m_{2}, u_{2}\right)$ be two TFN, then their operations are shown as follows (Kauffman and Gupta 1991):

$$
\begin{gathered}
A \oplus B^{\prime}=\left(l_{1}, m_{1}, u_{1}\right) \oplus\left(l_{2}, m_{2}, u_{2}\right)=\left(l_{1}+l_{2}, m_{1}+m_{2}, u_{1}+u_{2}\right) \\
\text { for } l_{i}>0, m_{i}>0, u_{i}>0, i=1,2
\end{gathered}
$$

$$
A<B=\left(l_{1}, m_{1}, u_{1}\right) \square\left(l_{2}, m_{2}, u_{2}\right)=\left(l_{1}-l_{2}, m_{1}-m_{2}, u_{1}-u_{2}\right)
$$

$A^{\prime} \otimes B^{\circ}=\left(l_{1}, m_{1}, u_{1}\right) \otimes\left(l_{2}, m_{2}, u_{2}\right)=\left(l_{1} l_{2}, m_{1} m_{2}, u_{1} u_{2}\right)$

$$
A q B^{\circ}=\left(l_{1}, m_{1}, u_{1}\right) /\left(l_{2}, m_{2}, u_{2}\right)=\left(l_{1} / u_{2}, m_{1} / m_{2}, u_{1} / u_{2}\right)
$$

$$
A^{-1}=\left(l_{1}, m_{1}, u_{1}\right)^{-1}=\left(l_{1} / u_{2}, m_{1} / m_{2}, u_{1} / u_{2}\right)
$$

\subsection{Distance of TFN}

Let $\tilde{A}=\left(l_{1}, m_{1}, u_{1}\right), \tilde{B}=\left(l_{2}, m_{2}, u_{2}\right)$, the distance between $\tilde{A}$ and $\tilde{B}$ is defined as follows (Van and Pedrycz 1983):

$$
d(A, B)=\frac{\left(l_{1}-l_{2}\right)+\left(m_{1}-m_{2}\right)+\left(u_{1}-u_{2}\right)}{3}
$$

\subsection{The extent analysis FAHP method}

The process of Chang's FAHP can be discussed as follows, the fuzzy pairwise comparison matrix $\tilde{A}=\left(\tilde{a}_{i j}\right)_{n \times n}$ can be mathematically defined as follow: 
where

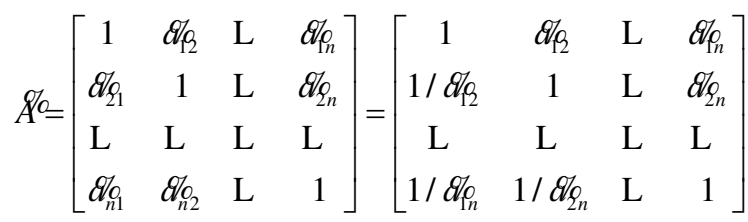

$$
\not=d_{i l}= \begin{cases}1 & i=j \\ 1,3,5,7,9 \text { or } \ldots 1^{-1}, 3^{-1}, 5^{-1}, 7^{-1}, 9^{-1} & i \neq j\end{cases}
$$

For $\mathrm{S}_{i}$, the fuzzy synthetic extent value, with respect to the $i$-th object is express as:

$$
S_{i}=\sum_{j=1}^{m} M_{i j} \otimes\left[\sum_{i=1}^{n} \sum_{j=1}^{m} M_{i j}\right]^{-1}
$$

With

$$
\begin{gathered}
\sum_{j=1}^{m} M_{i j}=\left(\sum_{j=1}^{m} l_{i j}, \sum_{j=1}^{m} m_{i j}, \sum_{j=1}^{m} u_{i j}\right), i=1,2, \ldots, n \\
\sum_{i=1}^{n} \sum_{j=1}^{m} M_{i j}=\left(\sum_{i=1}^{n} \sum_{j=1}^{m} l_{i j}, \sum_{i=1}^{n} \sum_{j=1}^{m} m_{i j}, \sum_{i=1}^{n} \sum_{j=1}^{m} u_{i j}\right) \\
{\left[\sum_{i=1}^{n} \sum_{j=1}^{m} M_{i j}\right]^{-1}=\left(\frac{1}{\sum_{i=1}^{n} \sum_{j=1}^{m} u_{i j}}, \frac{1}{\sum_{i=1}^{n} \sum_{j=1}^{m} m_{i j}}, \frac{1}{\sum_{i=1}^{n} \sum_{j=1}^{m} l_{i j}}\right)}
\end{gathered}
$$

The degree of possibility of $S_{j}=\left(l_{\mathrm{j}}, u_{\mathrm{j}}, m_{\mathrm{j}}\right) \geqslant S_{i}=\left(l_{i}, u_{i}, m_{i}\right)$ is denoted as:

$$
V\left(S_{j} \geq S_{i}\right)=\operatorname{height}\left(S_{i} \cap S_{j}\right)= \begin{cases}1 & \text { if } m_{j} \geq m_{i} \\ 0 & \text { if } l_{i} \geq u_{j} \\ \frac{l_{i}-u_{j}}{\left(m_{j}-u_{j}\right)-\left(m_{i}-l_{i}\right)} & \text { otherwise }\end{cases}
$$

To compare between $S_{j}$ and $S_{i}$, it is required to calculate both $V\left(S_{i} \geq S_{j}\right)$ and $V\left(S_{j} \geq S_{i}\right)$. The minimum degree of possibility $d(i)$ of $V\left(S_{j} \geq S_{i}\right)$ for $i, j=1,2, \ldots, k$ is calculated as follows (see Fig. 2):

$$
\begin{gathered}
\quad V\left(S \geq S_{1}, S_{2}, S_{3}, \mathrm{~L}, S_{k}\right) \\
=V\left[\left(S \geq S_{1}\right) \text { 和 }\left(S \geq S_{2}\right) \text { 和 } \mathrm{L}\left(S \geq S_{k}\right)\right] \\
=\min V\left(S \geq S_{i}\right), i=1,2,3, \mathrm{~L}, k
\end{gathered}
$$




$$
W=\left(d\left(A_{1}\right), d\left(A_{2}\right), \mathrm{L}, d\left(A_{n}\right)\right)^{T}
$$

188 Here $A_{i}(i=1,2, \ldots, n)$ are $n$ elements.

189 The weight vectors are obtained by normalizing as below:

190

$$
W=\left(W_{1}, W_{2}, \mathrm{~L}, W_{n}\right)^{T}
$$

191 Where $W$ is a real number.

192

193

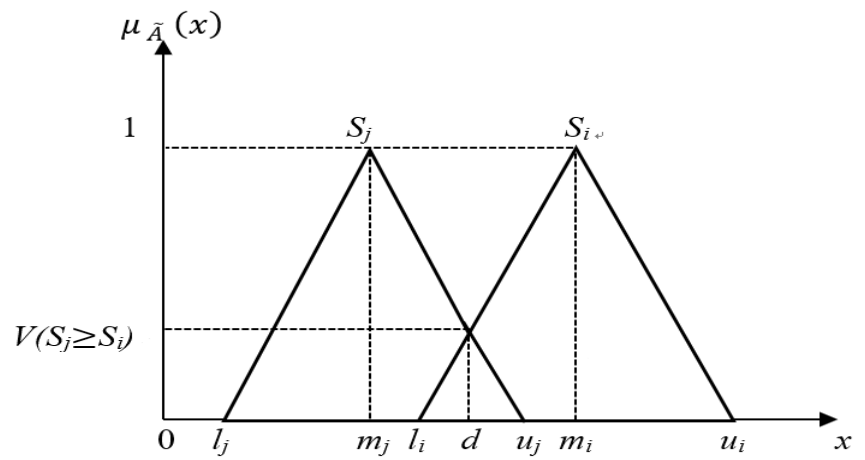

Fig. 2. The intersection between $\mathrm{S}_{j}$ and $\mathrm{S}_{i}$.

\subsection{Questionnaire design and linguistic scales}

In this study, TFN expresses a subjective pairwise comparison of decision makers. The linguistic terms and graphical representation of triangular linguistic labels are shown in Table 1 and Fig. 3 ((Kahraman et al. 2006)).

Table 1 Linguistic scales and fuzzy scales for importance.

\begin{tabular}{ccc}
\hline Linguistic scale for importance & Triangular fuzzy scale & $\begin{array}{c}\text { Triangular fuzzy reciprocal } \\
\text { scale }\end{array}$ \\
\hline Absolutely more important (AMI) & $(5 / 2,3,7 / 2)$ & $(2 / 7,1 / 3,2 / 5)$ \\
Very strongly more important (VSMI) & $(2,5 / 2,3)$ & $(1 / 3,2 / 5,1 / 2)$ \\
Strongly more important (SMI) & $(3 / 2,2,5 / 2)$ & $(2 / 5,1 / 2,2 / 3)$ \\
Weakly more important (WMI) & $(1,3 / 2,2)$ & $(1 / 2,2 / 3,1)$ \\
Equally important (EI) & $(1 / 2,1,3 / 2)$ & $(2 / 3,1,2)$ \\
Just equal & $(1,1,1)$ & $(1,1,1)$ \\
\hline
\end{tabular}

namely, the importance of one factor over another is divided into six levels 


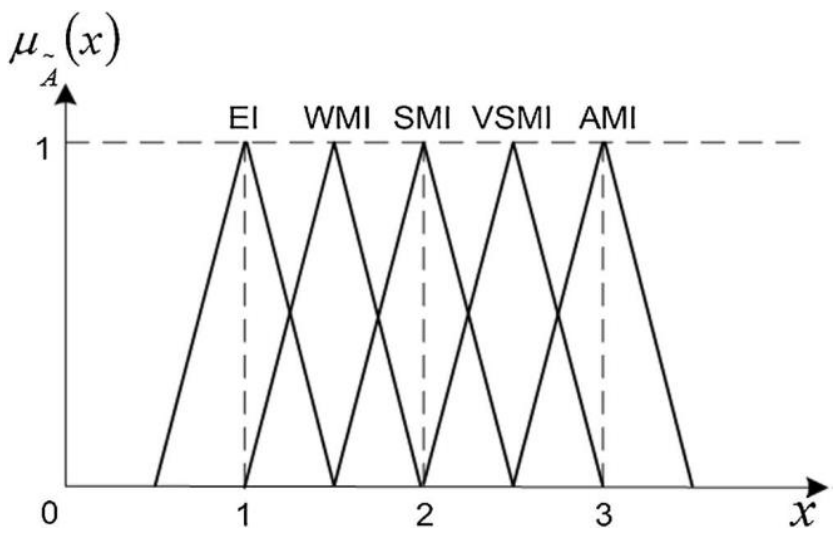

Fig 3. Linguistic scale for relative importance.

\subsection{Establishing the comparison matrix of the decision-makers}

Integration the opinions of multiple decision makers is one of the characteristics of the AHP. In the application of this method, geometric mean operation is commonly used to integrating group opinions, and this operation satisfies Pareto principle (unanimity condition) and homogeneity condition (Chen et al. 2015). This study is composed of K decision-makers (experts), who construct the group comparison matrix by compare $n$ factors in pairs. In the group comparison matrix, the TFNs can be expressed as:

$$
\begin{aligned}
& l_{i j}=\min \left(l_{i j k}\right),(k=1,2, \mathrm{~L}, K) \\
& m_{i j}=\sqrt{\prod_{k=1}^{K} m_{i j k}},(k=1,2, \mathrm{~L}, K) \\
& u_{i j}=\max \left(u_{i j k}\right),(k=1,2, \mathrm{~L}, K)
\end{aligned}
$$

As a result of this comparisons, a series of $k$ matrices, $\widetilde{A_{k}}=\left(\tilde{a}_{i j k}\right)$, can be obtained; where $\tilde{a}_{i j k}=\left(l_{i j}, m_{i j}, u_{i j}\right)$ denotes an importance of factor $i$ to $j$ relatively, as evaluated by the decision-maker $k$.

\subsection{The consistency tests}

In order to obtain rational assessment results, the consistency must be tested. Consequently, a fuzzy comparison matrix is transformed into a crisp matrix. In this study, Chang's approach is used to defuzzify a fuzzy number and the fuzzy perception can be expressed reasonably (Chang et al. 2009). A TFN represented as $\tilde{a}_{i j}=\left(l_{i j}, m_{i j}, u_{i j}\right)$ can be defuzzified to a crisp number as follows:

$$
\left(a_{i j}^{\alpha}\right)^{\lambda}=\left[\lambda . l_{i j}^{\alpha}+(1-\lambda) u_{i j}^{\alpha}\right], 0 \leq \lambda \leq 1,0 \leq \alpha \leq 1
$$

Where $l_{i j}^{\alpha}=\left(m_{i j}-l_{i j}\right) \times \alpha+l_{i j}$, is interpreted as the left-end value of $\alpha$-cut for $a_{i j}$. Conversely, $u_{i j}^{\alpha}=u_{i j}-\left(u_{i j}-m_{i j}\right) \times \alpha$ is interpreted as the right-end value of $\alpha$-cut. $\lambda$ indicates the degree of optimism among decision-makers, which is any numerical value from 0 to 1 [52]. When $\lambda$ is equal to 0 , the degree of optimism is maximum among 
decision-makers. Conversely, when $\lambda$ is equal to 1 , a decision-maker is highly pessimistic. Additionally, the decision-making circumstances fluctuating if $\alpha$ decreases. When $\alpha=0$, the degree of uncertainty is the maximum. Therefore, the above formula can be described as a fluctuating or stable condition, and its range is from 0 to 1 .

In a comparison matrix, all the factors of assessment model can be transformed from TFNs to crisp numbers (Hsu et al. 2016), which is shown as follows:

$$
\left[\left(A^{\alpha}\right)^{\lambda}\right]=\left[\left(a_{i j}\right)^{\lambda}\right]=\left[\begin{array}{cccc}
1 & \left(a_{12}^{\alpha}\right)^{\lambda} & \mathrm{L} & \left(a_{1 n}^{\alpha}\right)^{\lambda} \\
\left(a_{21}^{\alpha}\right)^{\lambda} & 1 & \mathrm{~L} & \left(a_{2 n}^{\alpha}\right)^{\lambda} \\
\mathrm{L} & \mathrm{L} & \mathrm{L} & \mathrm{L} \\
\left(a_{n 1}^{\alpha}\right)^{\lambda} & \left(a_{n 2}^{\alpha}\right)^{\lambda} & \mathrm{L} & 1
\end{array}\right]
$$

Where, superscripts $\alpha$ and $\lambda$ are for comparisons based on equation (19).

In order to check the consistency of the comparison matrix, the consistency index $(C I)$ and the consistency ratio $(C R)$ are given as [34]:

$$
\begin{gathered}
C I=\frac{\lambda_{\max }-n}{n-1} \\
C R=\frac{C I}{R I}
\end{gathered}
$$

Here $R I$ is the random index and $\lambda_{\max }$ denote the maximum eigenvalue. As is shown in Table 2, if $C R \leq 0.1$, the consistency of the judgment matrix is acceptable range.

Table 2 The random consistency index

\begin{tabular}{llllllllll}
\hline$n$ & 1 & 2 & 3 & 4 & 5 & 6 & 7 & 8 & 9 \\
\hline$R I$ & 0 & 0 & 0.58 & 0.09 & 1.12 & 1.24 & 1.32 & 1.41 & 1.45 \\
\hline
\end{tabular}

\section{Fuzzy comprehensive evaluation method}

FCE is an application of the fuzzy set theory. FCE is a quantitative, reasonable and objective evaluation method, suggested by Zadeh (Zadeh 1965; Zadeh 1978). Although FCE has been widely carried out in many areas, there are few utilizations of FCE in the field of the BBS management. In this paper, we used FCE as a tool for sustainable safety performance assessment and its application steps are as follows:

\subsection{Evaluation factor set}

$$
U=\left\{U_{m 1}, U_{m 2}, \ldots, U_{m p}\right\}
$$

Where $m$ denotes number of criteria layers, $p$ is the number of sub-criteria layers.

\subsection{Evaluation set}

$$
V=\left\{V_{1}, V_{2}, \ldots, V_{n}\right\}
$$

Evaluation set $V$ is consisted of different evaluation results. In this paper, $V$ is can be divided into $n$ subsets ( $n=5)$. $V_{1}, V_{2} V_{3} V_{4}$ and $V_{5}$ denote very low, low, medium, high, 
253 very high, respectively. They demonstrate the uncertainty and ambiguity of human 254 thinking.

\section{$255 \quad 3.3 \quad$ Determining a fuzzy relationship matrix}

256 In the evaluation factor set, a single-factor is evaluated to determine its membership 257 degree. The fuzzy relation matrix can be expressed as follows:

$$
R=\left(r_{i j}\right)_{m \times n}=\left[\begin{array}{cccc}
r_{11} & r_{12} & \mathrm{~K} & r_{1 n} \\
r_{21} & r_{22} & \mathrm{~K} & r_{2 n} \\
\mathrm{M} & \mathrm{M} & \mathrm{M} & \mathrm{M} \\
r_{m 1} & r_{m 2} & \mathrm{~K} & r_{m n}
\end{array}\right]
$$

where, $r_{i j}$ is the fuzzy membership degree result of the $i$ th factor belong to the $j$ th rank.

\subsection{Calculating fuzzy comprehensive evaluation results}

As described in section 4, the weight set $W$ is obtained by using FAHP. Therefore, the FCE model can be established based on weight set $W$ and fuzzy relationship matrix $R$ as follow:

$$
\begin{aligned}
B & =W \cdot R \\
& =\left(W_{1}, W_{2}, \mathrm{~L}, W_{m}\right)\left[\begin{array}{cccc}
r_{11} & r_{12} & \mathrm{~K} & r_{1 n} \\
r_{21} & r_{22} & \mathrm{~K} & r_{2 n} \\
\mathrm{M} & \mathrm{M} & \mathrm{M} & \mathrm{M} \\
r_{m 1} & r_{m 2} & \mathrm{~K} & r_{m n}
\end{array}\right] \\
& =\left(b_{1}, b_{2}, \mathrm{~L}, b_{n}\right)
\end{aligned}
$$

The results of the FCE can be obtained based on the maximum membership principle.

\section{The proposed framework}

BBS management is mainly used to improve the safety behaviors performance of employees. However, in the lack of a large amount of data, it is necessary to integrate expert judgments of experience into the safety assessment. In order to evaluate the safety of complex management system, a flexible and comprehensive technique method needs to be provided. Therefore, our proposed method integrates the FAHP, multi-criteria technique, fuzzy comprehensive evaluation with BBS management. This method integrates the group consistent decision-making principle, which can not only select the suitable alternative but also scientifically quantify the sustainable safety performance of employees. In Fig. 4, we describe a flowchart of the assessment framework and safety behaviors analysis based on the combined application of FAHP, FCE and BBS management. 


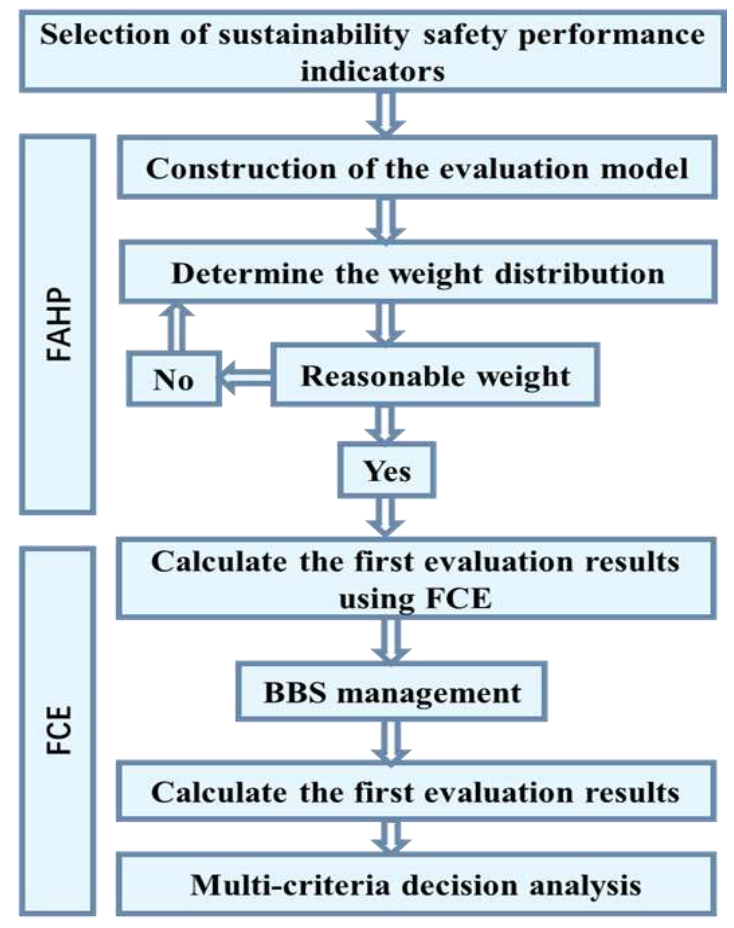

Fig. 4. The flowchart of the proposed assessment framework.

As illustrated, FAHP, FCE and BBS process are combined as a part of the safety management. The colored module shows the application of FAHP and FCE quantified safety behavior, which is implemented by the combination assessment of these two approaches.

More specifically, the FAHP is used to pairwise comparisons which are calculate the local and global priority values and prioritize the safety behaviors factors. Next, the evaluation grade is calculated, the conclusion of performance of employees' safety behavior is obtained by combining qualitative analysis and quantitative methods. According to the application of BBS program, the safety behavior of employees is guided and observed and intervene in the chemic plant. Finally, after a period of BBS process, the assessment grade is again calculated about sustainable safety performance.

\subsection{Application of proposed research framework}

The mathematical model has been applied to a state-owned petrochemical plant in China. This petrochemical plant includes an administrative leading department, facility management, petroleum processing department, quality control department, oil and gas gathering team, oil refining department, information management center, etc. Analyze the occupational injury statistics of this company, it is found that the accidents are mainly caused by human factors. Detailed analysis shows that about $90 \%$ of industry accidents are caused by employees' unsafe behaviors. In the high-risk operation system, it is of great significant to determine the safety behavior factors and take necessary 
preventive measures to reduce the accidents occupational injuries. Furthermore, with the help of an experienced scholar and administrator, 5 groups of 30 experienced managers, supervisor, scholars, engineers and employees have been discussed and adjusted the safety behavior factors of petrochemical plant. In consequence, the model is built by five group of experts as shown in Figure 5.

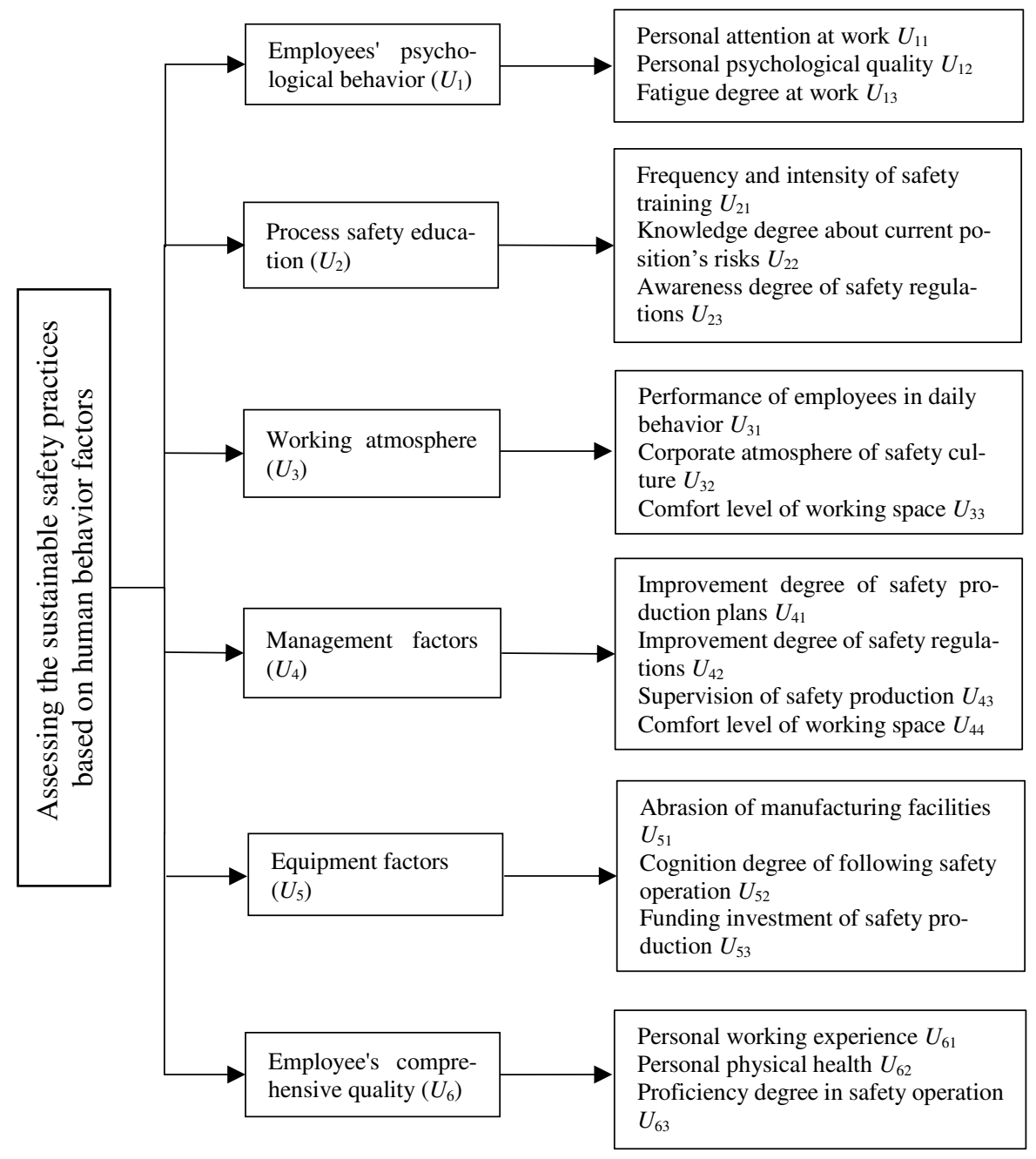

Fig. 5. The assessment model of sustainable safety performance

As shown in Fig. 5, the evaluation index system consists of three levels. The first level is the target layer. The second level is the criterion layer, which includes six factors $\left(U_{1}-U_{6}\right)$, such as employees' psychological behavior $\left(U_{1}\right)$, Process safety education $311\left(U_{2}\right)$, working atmosphere $\left(U_{3}\right)$, management factors $\left(U_{4}\right)$, equipment factors $\left(U_{5}\right)$ and 
312 employee's comprehensive quality $\left(U_{6}\right)$. The third level is the sub-criterion layer in-

313 cluding nineteen sub-factors $\left(U_{11}-U_{63}\right)$.

\section{$314 \quad 4.2$ Weights are calculated using FAHP}

315

316

317

318

319

320

321

To calculate the criterion weights, five groups of 30 experts are designated. The experts were organized for an in-depth interview, and asked to compare six criteria and 19 subcriteria in the evaluation model. Pairwise comparisons, sourced from the expert evaluations on the importance of one criterion over another relatively, are used to establish the comparison matrix of every expert. The geometric mean operation is utilized to obtain the representative comparison matrix of each expert group by Eq. (18). Three groups of factors of representative comparison matrix collected from the expert group are respectively shown in Tables 3 .

Table 3 Comparison matrix of the factors.

\begin{tabular}{lllllll}
\hline & $U_{1}$ & $U_{2}$ & $U_{3}$ & $U_{4}$ & $U_{5}$ & $U_{6}$ \\
\hline$U_{1}$ & $(1,1,1)$ & $(0.5,1.18,2)$ & $(0.5,1.2,3)$ & $(0.29,1.13,3)$ & $(0.29,1.2,3)$ & $(0.5,1.97,3.5)$ \\
$U_{2}$ & $(0.5,0.69,2)$ & $(1,1,1)$ & $(0.33,0.9,2)$ & $(0.33,1.29,3)$. & $(0.5,0.91,3)$ & $(0.5,1.55,3.5)$ \\
$U_{3}$ & $(0.33,0.83,2)$ & $(0.5,1.08,3)$ & $(1,1,1)$ & $(0.67,1.55,3)$. & $(0.33,0.77,3)$ & $(0.5,1.46,3.5)$ \\
$U_{4}$ & $(0.33,0.88,3)$. & $(0.5,0.8,3.5)$ & $(0.5,0.64,1.5)$ & $(1,1,1)$ & $(0.5,1.19,3.5)$ & $(0.67,1.38,2)$ \\
$U_{5}$ & $(0.33,0.83,3)$. & $(0.33,1.1,2.5)$ & $(0.3333,1.3)$, & $(0.29,0.84,0)$. & $(1,1,1)$ & $(0.5,1.21,1)$ \\
$U_{6}$ & $(0.29,0.51,2)$ & $(0.29,0.8,2)$ & $(0.29,0.99,3)$ & $(0.5,0.72,1.5)$ & $(0.4,0.82,2)$ & $(1,1,1)$ \\
\hline
\end{tabular}

According to the environmental and conditional uncertainty, the experts can decide the $\alpha$-cut subjectivity. In this paper, $\alpha=0.5$ is used to expresses that conditional and environmental uncertainty is stabilized, and $\lambda=0.5$ denotes that the attitude of the experts is fair and rational. When $\alpha=0.5, \lambda=0.5$, the defuzzification of in Table 3 is expressed as follows:

$$
\begin{gathered}
l_{12}^{0.5}=(1.1761-0.5) \times 0.5+0.5=0.8381 \\
u_{12}^{0.5}=2-(2-1.1761) \times 0.5=1.5881 \\
\left(a_{12}^{0.5}\right)^{0.5}=[0.5 \times 0.8381+(1-0.5) \times 1.5881]=1.2131
\end{gathered}
$$

Similar to the calculation above, the crisp comparison matrix of all factors is obtained in Table 4,

Table 4 Crisp comparison matrix of the factors

\begin{tabular}{lllllll}
\hline & $U_{1}$ & $U_{2}$ & $U_{3}$ & $U_{4}$ & $U_{5}$ & $U_{6}$ \\
\hline$U_{1}$ & 1.0000 & 1.2131 & 1.4756 & 1.3885 & 1.4220 & 1.9872 \\
$U_{2}$ & 0.8243 & 1.0000 & 1.1181 & 1.4399 & 1.3302 & 1.7760 \\
$U_{3}$ & 0.6777 & 0.8944 & 1.0000 & 1.8176 & 1.2172 & 1.7307 \\
$U_{4}$ & 0.7202 & 0.6945 & 0.5502 & 1.0000 & 1.5957 & 1.3583 \\
$U_{5}$ & 0.7032 & 0.7518 & 0.8216 & 0.6267 & 1.0000 & 1.1084 \\
$U_{6}$ & 0.5032 & 0.5631 & 0.5778 & 0.7362 & 0.9022 & 1.0000 \\
\hline
\end{tabular}

The next step is to calculate the consistency of the crisp comparison matrix. Firstly, by using Eqs. (21) and (22), $C R=0.0108<0.1$. This shows that the crisp comparison matrix satisfies consistency and the weight distribution is reasonable. 
We use Chang's extent analysis method and the fuzzy evaluation matrix of six factors $\left(U_{1}-U_{6}\right)$ in Table 3 to calculate the factors weights. Using Eq. (10) - (14) to calculate the value of fuzzy synthetic extent, the factors and sub-factor weights is computed by the FAHP. Taking the factor weights of the pairwise comparison matrix in Table 3 as an example, the value of fuzzy synthetic extent is calculated as follow:

$$
\begin{gathered}
S_{1}=(3.071,7.687,15.5) \otimes\left(\frac{1}{81.9}, \frac{1}{37.751}, \frac{1}{18.61}\right)=(0.038,0.204,0.833) \\
S_{2}=(3.167,6.34,15) \otimes\left(\frac{1}{81.9}, \frac{1}{37.751}, \frac{1}{18.61}\right)=(0.043,0.156,0.806) \\
S_{3}=(3.333,6.69,16) \otimes\left(\frac{1}{81.9}, \frac{1}{37.751}, \frac{1}{18.61}\right)=(0.039,0.168,0.806) \\
S_{4}=(3.5,5.904,15) \otimes\left(\frac{1}{81.9}, \frac{1}{37.751}, \frac{1}{18.61}\right)=(0.034,0.167,0.478) \\
S_{5}=(2.786,6.29,8.9) \otimes\left(\frac{1}{81.9}, \frac{1}{37.751}, \frac{1}{18.61}\right)=(0.041,0.177,0.860) \\
S_{6}=(2.757,4.841,11.5) \otimes\left(\frac{1}{81.9}, \frac{1}{37.751}, \frac{1}{18.61}\right)=(0.034,0.128,0.618)
\end{gathered}
$$

The degree of possibility of every factor over the others is calculated by using Eqs. (15), which results are shown as follow:

$$
\begin{gathered}
\mathrm{V}\left(\mathrm{S}_{1} \geq \mathrm{S}_{2}\right)=1, \mathrm{~V}\left(\mathrm{~S}_{1} \geq \mathrm{S}_{3}\right)=1, \mathrm{~V}\left(\mathrm{~S}_{1} \geq \mathrm{S}_{4}\right)=1, \mathrm{~V}\left(\mathrm{~S}_{1} \geq \mathrm{S}_{5}\right)=1, \mathrm{~V}\left(\mathrm{~S}_{1} \geq \mathrm{S}_{6}\right)=1 \\
\mathrm{~V}\left(\mathrm{~S}_{2} \geq \mathrm{S}_{1}\right)=\frac{l_{1}-u_{2}}{\left(m_{2}-u_{2}\right)-\left(m_{1}-l_{1}\right)}=\frac{0.038-0.806}{(0.156-0.806)-(0.204-0.038)}=0.9418, \mathrm{~V}\left(\mathrm{~S}_{2} \geq \mathrm{S}_{3}\right) \\
\begin{array}{r}
0.9846, \\
\mathrm{~V}\left(\mathrm{~S}_{2} \geq \mathrm{S}_{4}\right)=0.986, \mathrm{~V}\left(\mathrm{~S}_{2} \geq \mathrm{S}_{5}\right)=0.9721, \mathrm{~V}\left(\mathrm{~S}_{2} \geq \mathrm{S}_{6}\right)=1 . \\
\mathrm{V}\left(\mathrm{S}_{3} \geq \mathrm{S}_{1}\right)=0.9552, \mathrm{~V}\left(\mathrm{~S}_{3} \geq \mathrm{S}_{2}\right)=1, \mathrm{~V}\left(\mathrm{~S}_{3} \geq \mathrm{S}_{4}\right)=1, \mathrm{~V}\left(\mathrm{~S}_{3} \geq \mathrm{S}_{5}\right)=0.9884, \mathrm{~V}\left(\mathrm{~S}_{3} \geq \mathrm{S}_{6}\right) \\
\quad=1 .
\end{array} \\
\begin{array}{r}
\mathrm{V}\left(\mathrm{S}_{4} \geq \mathrm{S}_{1}\right)=0.9224, \mathrm{~V}\left(\mathrm{~S}_{4} \geq \mathrm{S}_{2}\right)=1, \mathrm{~V}\left(\mathrm{~S}_{4} \geq \mathrm{S}_{3}\right)=0.9977, \mathrm{~V}\left(\mathrm{~S}_{4} \geq \mathrm{S}_{5}\right)=0.9776, \mathrm{~V}\left(\mathrm{~S}_{4}\right. \\
\left.\quad \mathrm{S} \mathrm{S}_{6}\right)=1 .
\end{array} \\
\mathrm{V}\left(\mathrm{S}_{5} \geq \mathrm{S}_{1}\right)=0.9364, \mathrm{~V}\left(\mathrm{~S}_{5} \geq \mathrm{S}_{2}\right)=1, \mathrm{~V}\left(\mathrm{~S}_{5} \geq \mathrm{S}_{3}\right)=1, \mathrm{~V}\left(\mathrm{~S}_{5} \geq \mathrm{S}_{4}\right)=1, \mathrm{~V}\left(\mathrm{~S}_{5} \geq \mathrm{S}_{6}\right)=1 . \\
\mathrm{V}\left(\mathrm{S}_{6} \geq \mathrm{S}_{1}\right)=0.8842, \mathrm{~V}\left(\mathrm{~S}_{6} \geq \mathrm{S}_{2}\right)=0.9536, \mathrm{~V}\left(\mathrm{~S}_{6} \geq \mathrm{S}_{3}\right)=0.9354, \mathrm{~V}\left(\mathrm{~S}_{6} \geq \mathrm{S}_{4}\right)=0.9374 \\
\mathrm{~V}\left(\mathrm{~S}_{6} \geq \mathrm{S}_{5}\right)=0.9217 .
\end{gathered}
$$

Thereafter, identifying the minimum degree of possibility $d(\mathrm{i})$ of $V\left(S_{i} \geq S_{j}\right)$ for $i, j=1$, $2, \ldots, 6$ by using Eq. (16), we have

$$
\begin{aligned}
& d^{\prime}(1)=\min V\left(S_{1} \geq S_{2}, S_{3}, S_{4}, S_{5}, S_{6}\right)=1.0000 \\
& d^{\prime}(2)=\min V\left(S_{2} \geq S_{1}, S_{3}, S_{4}, S_{5}, S_{6}\right)=0.9721 \\
& d^{\prime}(3)=\min V\left(S_{3} \geq S_{1}, S_{2}, S_{4}, S_{5}, S_{6}\right)=0.9552 \\
& d^{\prime}(4)=\min V\left(S_{4} \geq S_{1}, S_{2}, S_{3}, S_{5}, S_{6}\right)=0.9224 \\
& d^{\prime}(5)=\min V\left(S_{5} \geq S_{1}, S_{2}, S_{3}, S_{4}, S_{6}\right)=0.9364 \\
& d^{\prime}(6)=\min V\left(S_{6} \geq S_{1}, S_{2}, S_{3}, S_{4}, S_{5}\right)=0.8842
\end{aligned}
$$

Therefore, based on Eqs. (16), the weight vector $W$ can be obtained as:

$$
W=(1,0.9721,0.9552,0.9224,0.9364,0.8842)^{T}
$$

The weight vector is normalized by using Eq. (17). The normalized weights vectors of the six factors are calculated as follow: 
Table 8 Comparison matrix of the "Management factors".

\begin{tabular}{lllll}
\hline & $U_{41}$ & $U_{42}$ & $U_{43}$ & $U_{44}$ \\
\hline$U_{41}$ & $1,1,1$ & $0.5,1.5281,2.5$ & $0.5,1.5281,3.5$ & $0.5,1.5646,3$ \\
$U_{42}$ & $0.3333,0.6544,2$ & $1,1,1$ & $0.4,0.9221,2.5$ & $0.3333,1.0592,3$ \\
$U_{43}$ & $0.2857,0.9221,3.5$ & $0.4,0.9221,2.5$ & $1,1,1$ & $1,2.4082,3.5$ \\
$U_{44}$ & $0.3333,0.6392,2$ & $0.3333,0.9441,3$ & $0.2857,0.4152,1$ & $1,1,1$ \\
\hline
\end{tabular}

$W=\left(W_{\mathrm{U} 1}, W_{\mathrm{U} 2}, W_{\mathrm{U} 3}, W_{\mathrm{U} 4}, W_{\mathrm{U} 5}, W_{\mathrm{U} 6}\right)^{T}=(0.1764,0.1714,0.1685,0.1627,0.1651$, $0.1559)^{T}$

Similarly, the weight vectors $W_{1}, W_{2}, W_{3}, W_{4}, W_{5}, W_{6}$ of sub-factors are calculated shown as below:

$$
\begin{gathered}
W_{1}=\left(W_{\mathrm{U} 11}, W_{\mathrm{U} 12}, W_{\mathrm{U} 13}\right)=[0.3462,0.3496,0.3043]^{T} \\
W_{2}=\left(W_{\mathrm{U} 21}, W_{\mathrm{U} 22}, W_{\mathrm{U} 23}\right)=[0.3896,0.2972,0.3132]^{T} \\
W_{3}=\left(W_{\mathrm{U} 31}, W_{\mathrm{U} 32}, W_{\mathrm{U} 33}\right)=[0.3640,0.3398,0.2961]^{T} \\
W_{4}=\left(W_{\mathrm{U} 41}, W_{\mathrm{U} 42}, W_{\mathrm{U} 43}, W_{\mathrm{U} 44}\right)=[0.2787,0.2460,0.2462,0.2290]^{T} \\
W_{5}=\left(W_{\mathrm{U} 51}, W_{\mathrm{U} 52}, W_{\mathrm{U} 53}\right)=[0.3532,0.3097,0.3371]^{T} \\
W_{6}=\left(W_{\mathrm{U} 61}, W_{\mathrm{U} 62}, W_{\mathrm{U} 63}\right)=[0.3614,0.3129,0.3257]^{T}
\end{gathered}
$$

Then, these experts construct to the pairwise comparisons of the relative importance of two sub-factors. These pairwise comparison matrices collected from five groups of 30 experts were shown in Tables 5-10.

Table 5 Comparison matrix of the "Employees' psychological behavior".

\begin{tabular}{llll}
\hline & $U_{11}$ & $U_{12}$ & $U_{13}$ \\
\hline$U_{11}$ & $1,1,1$ & $0.5,0.8503,2$ & $0.5,1.431,2.5$ \\
$U_{12}$ & $0.5,0.8503,2$ & $1,1,1$ & $1,1.4963,3$ \\
$U_{13}$ & $0.4,0.6988,2$ & $0.3333,0.7677,2$ & $1,1,1$ \\
\hline
\end{tabular}

Table 6 Comparison matrix of the "Safety education".

\begin{tabular}{llll}
\hline & $U_{21}$ & $U_{22}$ & $U_{23}$ \\
\hline$U_{21}$ & $1,1,1$ & $1,1.5889,2$ & $0.3333,1.6438,3.5$ \\
$U_{22}$ & $0.4,0.6294,1$ & $1,1,1$ & $0.5,0.6294,3$ \\
$U_{23}$ & $0.2857,0.6084,3$ & $0.3333,0.7079,2$ & $1,1,1$ \\
\hline
\end{tabular}

Table 7 Comparison matrix of the "Working atmosphere".

\begin{tabular}{llll}
\hline & $U_{31}$ & $U_{32}$ & $U_{33}$ \\
\hline$U_{31}$ & $1,1,1$ & $0.4,1.1487,2.5$ & $0.6667,1.7972,3.5$ \\
$U_{32}$ & $0.4,0.8706,2.5$ & $1,1,1$ & $0.5,1.3797,3$ \\
$U_{33}$ & $0.3333,0.5564,2$ & $0.4,0.7248,2$ & $1,1,1$ \\
\hline
\end{tabular}

Table 9 Comparison matrix of the "Equipment factors". 


\begin{tabular}{llll}
\hline$U_{51}$ & $1,1,1$ & $0.3333,1.292,3.5$ & $1,1.2619,3.5$ \\
$U_{52}$ & $0.2857,0.774,3$ & $1,1,1$ & $0.4,0.7579,2$ \\
$U_{53}$ & $0.2857,0.7925,3$ & $0.5,1.3195,2.5$ & $1,1,1$ \\
\hline
\end{tabular}

Table 10 Comparison matrix of the "Employee's comprehensive quality".

\begin{tabular}{llll}
\hline & $U_{61}$ & $U_{62}$ & $U_{63}$ \\
\hline$U_{61}$ & $1,1,1$ & $0.3333,0.9872,2.5$ & $1,1.7597,3$ \\
$U_{62}$ & $0.3333,1.2011,1$ & $1,1,1$ & $0.3333,0.7248,2$ \\
$U_{63}$ & $0.3333,0.5682,1$ & $0.5,1.3797,3$ & $1,1,1$ \\
\hline
\end{tabular}

By a similar operation, the weight vectors of sub-factors were determined. Finally, all of the $C R \leqslant 0.1$, the consistency in each crisp comparison matrix is accepted.

\subsection{The BBS program for a petrochemical plant}

As more and more managers realize the importance of BBS process, many Chinese companies have also implemented BBS procedures in their management process (Chen and Tian 2012; Zhang et al. 2017; Yu and Li 2019). Hence, to better understand the BBS practices to Chinese company, this paper selected a state-owned petrochemical plant in the east of China as a study case. The specific BBS program is shown as follow:

\begin{tabular}{|c|c|c|c|c|}
\hline $\begin{array}{c}\text { Preparation } \\
\text { phase }\end{array}$ & $\begin{array}{l}\text { Design } \\
\text { phase }\end{array}$ & $\begin{array}{c}\text { Implementation } \\
\text { phase }\end{array}$ & $\begin{array}{c}\text { Intervention } \\
\text { phase }\end{array}$ & $\begin{array}{c}\text { Follow-up } \\
\text { phase }\end{array}$ \\
\hline$\downarrow$ & $\downarrow$ & $\downarrow$ & $\downarrow$ & $\downarrow$ \\
\hline $\begin{array}{l}\text { - Organizational } \\
\text { structure } \\
\text { - Plant history } \\
\text { - Personnel } \\
\text { - Management } \\
\text { system }\end{array}$ & $\begin{array}{l}\text { - Design team } \\
\text { - Observation } \\
\text { training } \\
\text { - Developed } \\
\text { checklists }\end{array}$ & $\begin{array}{l}\text { - Observation team } \\
\text { - Safety training } \\
\text { - Program launched }\end{array}$ & $\begin{array}{l}\text { - Safety } \\
\text { communication } \\
\text { - Information } \\
\text { feedback } \\
\text { - Safety maintenance } \\
\text { - Safety interaction }\end{array}$ & $\begin{array}{l}\text { - Observation } \\
\text { - Feedback } \\
\text { - Training } \\
\text { - Reward }\end{array}$ \\
\hline
\end{tabular}

Fig. 6. The flowchart of BBS program.

As illustrated, there are five steps in the BBS program, including the preparation period, design period, implementation period, intervention period, follow-up period. In addition, detailed process includes expert interview, questionnaire design, collection of data and assessment of sustainable safety performance and so on.

\section{Preparation Period}

Firstly, in the BBS process, the first step is to assess the sustainable safety performance of employees, understand the current status of the petrochemical plant and review the existing organizational structure, plant history, personnel, existing safety management system. Therefore, the existing employee of the petrochemical plant were questionnaire surveyed on the sustainable safety performance in Fig. 5. Likert five-point scale is used 
418 to collect the questionnaire data (Likert, 1932). In the first survey, a total of 824 ques419 tionnaires were received and 665 were available. Based on the questionnaire data, the 420 fuzzy relationship matrix is obtained by using Eq (24):

Where $R A_{1}, R A_{2}, \ldots, R A_{6}$ denote the fuzzy relationship matrix of the first assessment.

$$
\begin{array}{r}
R A_{1}=\left[\begin{array}{lllll}
0.0088 & 0.0380 & 0.3158 & 0.6199 & 0.0175 \\
0.0307 & 0.0775 & 0.4284 & 0.4415 & 0.0219 \\
0.0219 & 0.0190 & 0.0673 & 0.3538 & 0.5380
\end{array}\right] \\
R A_{2}=\left[\begin{array}{lllll}
0.0219 & 0.0365 & 0.0731 & 0.3421 & 0.5263 \\
0.0146 & 0.0234 & 0.0658 & 0.4269 & 0.4693 \\
0.0146 & 0.0146 & 0.1213 & 0.4854 & 0.3640
\end{array}\right]
\end{array}
$$$$
R A_{3}=\left[\begin{array}{llllll}
0.0029 & 0.0029 & 0.1067 & 0.4415 & 0.4459 \\
0.0073 & 0.0205 & 0.0731 & 0.4722 & 0.4269 \\
0.0058 & 0.0175 & 0.1360 & 0.5673 & 0.2734
\end{array}\right]
$$$$
R A_{4}=\left[\begin{array}{llllll}
0.0205 & 0.0468 & 0.0234 & 0.3772 & 0.5322 \\
0.0044 & 0.0058 & 0.0994 & 0.5365 & 0.3538 \\
0.0263 & 0.0117 & 0.1491 & 0.4795 & 0.3333 \\
0.0073 & 0.0073 & 0.1520 & 0.5804 & 0.2529
\end{array}\right]
$$$$
R A_{5}=\left[\begin{array}{llllll}
0.0014 & 0.0072 & 0.0807 & 0.4640 & 0.4467 \\
0.0029 & 0.0088 & 0.0863 & 0.5322 & 0.3699 \\
0.0058 & 0.0058 & 0.0658 & 0.4576 & 0.4649
\end{array}\right]
$$$$
R A_{6}=\left[\begin{array}{lllll}
0.0044 & 0.0029 & 0.0687 & 0.5336 & 0.3904 \\
0.0249 & 0.0161 & 0.0570 & 0.4576 & 0.4444 \\
0.0234 & 0.0058 & 0.0307 & 0.5058 & 0.4342
\end{array}\right]
$$

Taking the fuzzy relationship matrix $R A_{1}$ as an example, when "Corporate frequency and intensity of safety training" was considered, $64.51 \%$ of employees rated it "very high", $31.88 \%$ of employees rated it "high", $3.46 \%$ rated it "medium", $0 \%$ rated it "low" and $0.15 \%$ rated it "very low".

Then, the first-layer fuzzy comprehensive evaluation result is obtained based on Eq. (26):

$$
F A_{1}=W_{U 1} \cdot R A_{1}=
$$

$$
\begin{aligned}
& (0.3462,0.3496,0.3043) \cdot\left[\begin{array}{lllll}
0.0088 & 0.0380 & 0.3158 & 0.6199 & 0.0175 \\
0.0307 & 0.0775 & 0.4284 & 0.4415 & 0.0219 \\
0.0219 & 0.0190 & 0.0673 & 0.3538 & 0.5380
\end{array}\right]
\end{aligned}
$$

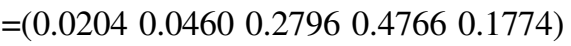

435 Similar to the $F A_{l}$ calculation, we get: 


$$
F A=\left[\begin{array}{llllll}
0.0204 & 0.0460 & 0.2796 & 0.4766 & 0.1774 \\
0.0174 & 0.0257 & 0.0860 & 0.4122 & 0.4585 \\
0.0053 & 0.0132 & 0.1039 & 0.4891 & 0.3883 \\
0.0149 & 0.0190 & 0.1025 & 0.4881 & 0.3753 \\
0.0033 & 0.0072 & 0.0774 & 0.4830 & 0.4291 \\
0.0170 & 0.0080 & 0.0527 & 0.5008 & 0.4216
\end{array}\right]
$$

According to Eq. (26) and the factors weights value, the assessment result set of second438 layer fuzzy comprehensive is:

$$
B_{1}=W \cdot F A=
$$

$(0.1764,0.1714,0.1685,0.1627,0.1651,0.1559)$

$\left[\begin{array}{llllll}0.0204 & 0.0460 & 0.2796 & 0.4766 & 0.1774 \\ 0.0174 & 0.0257 & 0.0860 & 0.4122 & 0.4585 \\ 0.0053 & 0.0132 & 0.1039 & 0.4891 & 0.3883 \\ 0.0149 & 0.0190 & 0.1025 & 0.4881 & 0.3753 \\ 0.0033 & 0.0072 & 0.0774 & 0.4830 & 0.4291 \\ 0.0170 & 0.0080 & 0.0527 & 0.5008 & 0.4216\end{array}\right]$

Where $B_{1}$ the fuzzy comprehensive results of the first evaluation. The results reveal that the probability of the sustainable safety performance very high is 0.3729 , the probability of 'high', 'medium', 'low' and 'very low' are 0.4744, 0.1192, 0.0203 and 0.0131, respectively. The result showing that the sustainable safety performance is assessed as high based the maximum membership principle. However, the employees' sustainable safety performance can be further improved in the future.

Table 11 Safety observation categories and checklist.

\begin{tabular}{cc}
\hline Categories & Description of the categories \\
\hline $\begin{array}{c}\text { Personal protective } \\
\text { equipment }\left(S O_{1}\right)\end{array}$ & Workers wear safety helmets at the work site \\
& Workers wear goggles when using chemicals \\
& Workers put on respiratory protection devices in dusty condi- \\
tions
\end{tabular}


Keep fire extinguishers in place

Traffic management Workers are not allowed to walk in the blind area of vehicle $\left(\mathrm{SO}_{2}\right)$

$$
\text { operators }
$$

The driver backed up under the guidance of the traffic dis-

patcher

Workers keep an eye on the coming traffic before crossing the street

Psychological performance $\left(\mathrm{SO}_{3}\right)$

Facilities maintenance $\left(\mathrm{SO}_{4}\right)$

Daily performance $\left(\mathrm{SO}_{5}\right)$
Maintain communication between supervisor and operator

$$
\text { Lazy attitude }
$$

Overconfidence

Workers wear safety belts when at work high above the ground Workers use a working platform without the risk of tripping or

$$
\text { falling }
$$

Workers do not use damaged ladders

Workers do not carry anything when up and down the ladder Operation areas of the machine have a clear cordoned off.

The operator shuts down the equipment before repairing Comply with the principles of hazardous waste disposal and

$$
\text { storage }
$$

Identifying damaged gaskets

Employees turn off unused lights and equipment

Worker takes the correct operation posture

Workers use appropriate access and egress

Workers are not allowed to discard anything from a height A rough assessment of the safety behaviour of workers on site

Five different behavior factors (coded as $\mathrm{SO}_{1}-\mathrm{SO}_{5}$ ) are used to observe the safety behaviors performance of on-site employees, including personal protective equipment $\left(\mathrm{SO}_{1}\right)$, traffic management $\left(\mathrm{SO}_{2}\right)$, psychological performance $\left(\mathrm{SO}_{3}\right)$, facilities maintenance $\left(\mathrm{SO}_{4}\right)$ and daily performance $\left(\mathrm{SO}_{5}\right)$. Based on the above factors, the checklist includes 23 typical employee's behaviors, which can be classified by $\mathrm{SO}_{1}-\mathrm{SO}_{5}$

\section{Implementation period}

The design team elect 35 experiences employees to set up the observation team. The role of the observation team is to maintain and monitor possible incidents during the BBS process. The observation team was received a two-day safety training (ST) course in the fundamental principle and practice of BBS management. The training course included decision making, unsafe behavior modification, how to manage others' resistance, observational and communicational skills, the provide personal feedback and effective checklist scoring (Dağdeviren and Yüksel 2008; Choudhry 2014; Wang et al. 2017). Safety training is as shown in Table 12. 
Table 12 Safety training categories and checklists.

\begin{tabular}{ll}
\hline Items & Specific content \\
\hline Safety communication $\left(S T_{1}\right)$ & The safety team having positive communicates with \\
& operators on site \\
& Actively participate in safety dialogue \\
& Increase communication with technicians on site \\
& Increase the frequency of informal conversations \\
& Report of attempted accidents \\
& Improve the efficiency of the meetings \\
Information feedback $\left(S T_{2}\right)$ & Improve the recognition of the meeting \\
& Reasonable time management \\
& Provide effective feedback \\
& Improve traffic safety \\
Safety maintenance $\left(S T_{3}\right)$ & Fill out work permits correctly \\
& Improve the quality of work \\
& Improve traffic safety \\
Safety interaction $\left(S T_{4}\right)$ & Increase communication with other sites \\
& Improve interaction between employees \\
& Ask safety questions on site \\
& Shaping safety behaviour \\
\hline
\end{tabular}

The Table 12 shows that the four-module course (coded as $S T_{1}-S T_{4}$ ) is designed to train employees in the petrochemical plant. After the training period, the design team held a plant-wide launch activity to announce the BBS process. Everyone attends a one-day training meeting on guiding observations and providing feedback. Then, everyone was trained on how to make observations and convey feedback to their colleagues. Various training meeting ensure that each employee know their role in the BBS process. When the BBS program was launched, employees began using both checklists for peer and self-observations.

\section{Intervention Period}

Administrative leadership system

Administrative leadership system is one of the characteristics of China's state-owned company and it can improve the willingness that everyone will accept the safety intervention, participate in observation, and provide individual feedback. The administrative leaders require managers and supervisors to regularly check the employee discipline, the participation in the BBS process, and maintenance of operation tools and equipment. Some leaders are also required to monitor the completion of the BBS process. The BBS management is implemented show that employees need support from their administrative leadership. In addition, these measures solve problems such as lack of communication in BBS process, low conference attendance and participation in observation (Brandhorst and Kluge 2021). 
492

\section{Observation}

To ensure authentic and objective safety participation for employees, all observations are voluntary. Everyone is invited to participate in peer safety observations or self-observations about 14 days. In addition, the BBS process requires each employee to complete two observations per day. The supervisors randomly observe the monitoring video of each employee every day for about 10-15 minutes. Supervisors and managers encourage their employees to spend the time to fulfil the observation, which can either be peer safety observations or self-observations. In every observation, the observers need to check all items in the safety behavior checklist and collect a behavior sample from each on-site employee to confirm whether it is safe or not. One receives a mental or a material reward for positively participating and supporting the BBS process, such as appreciation from a supervisor.

\section{Feedback}

BBS process studies have shown that safety behavior improves when management provides clear feedback on employee observation information (Favero et al. 2016). Therefore, safety observation team inspects information on participation and observation every day, such as use video monitoring to recognize the unsafe behavior or potential hazards. Then, by investigating the situation on the ground and working with corresponding management to deal with any observed unsafe behavior or equipment problems. Furthermore, an observation report meeting is held twice a day; One is before the employees starts work, and the other is before the employees leave work. The observation results are feed back to the employees on site during the report meeting on every day. Five working groups were identified as the highest rate of unsafe behavior. Employees of the five groups were asked to attend specific training courses such as watch accident videos. The goal of training courses is to improve their safety behavior and safety awareness, not punish these employees.

\section{Follow-Up Period}

At the end of the intervention phase, the questionnaire used in the preparation period were again distributed to the employees and to evaluate the changes of their safety behaviors. During this period, employees' behaviors were continuously observed and intervened. In the second survey, a total of 853 questionnaires have been collected, among which 726 replies were usable, with an effective replies rate of $85.11 \%$. The results of the twice assessment is contrast, and the scientific of the integration of the FAHP, BBS approach and FCE can be proved by the following evaluation. The second FCE results of sustainable safety performance from employees are as follows.

$$
\begin{array}{r}
R B_{1}=\left[\begin{array}{lllll}
0.0000 & 0.0000 & 0.0413 & 0.3048 & 0.6538 \\
0.0000 & 0.0000 & 0.0171 & 0.5128 & 0.4701 \\
0.0000 & 0.0000 & 0.0798 & 0.4345 & 0.4858
\end{array}\right] \\
R B_{2}=\left[\begin{array}{lllll}
0.0000 & 0.0000 & 0.0798 & 0.3718 & 0.5484 \\
0.0000 & 0.0014 & 0.0726 & 0.4786 & 0.4473 \\
0.0057 & 0.0014 & 0.1239 & 0.4373 & 0.4316
\end{array}\right]
\end{array}
$$




$$
\begin{array}{r}
R B_{3}=\left[\begin{array}{lllll}
0.0014 & 0.0028 & 0.0769 & 0.3989 & 0.5199 \\
0.0000 & 0.0014 & 0.0798 & 0.3989 & 0.5199 \\
0.0000 & 0.0085 & 0.0442 & 0.4088 & 0.5385
\end{array}\right] \\
R B_{4}=\left[\begin{array}{lllll}
0.0000 & 0.0000 & 0.0185 & 0.2635 & 0.7179 \\
0.0000 & 0.0000 & 0.0527 & 0.5342 & 0.4131 \\
0.0043 & 0.0014 & 0.0313 & 0.5370 & 0.4259 \\
0.0028 & 0.0043 & 0.0228 & 0.5442 & 0.4259
\end{array}\right]
\end{array}
$$

$$
R B_{5}=\left[\begin{array}{lllll}
0.0014 & 0.0000 & 0.1311 & 0.3903 & 0.4772 \\
0.0000 & 0.0000 & 0.1068 & 0.4416 & 0.4516 \\
0.0028 & 0.0014 & 0.0598 & 0.4644 & 0.4715
\end{array}\right]
$$$$
R B_{6}=\left[\begin{array}{lllll}
0.0014 & 0.0000 & 0.0584 & 0.5399 & 0.4003 \\
0.0000 & 0.0000 & 0.0655 & 0.3860 & 0.5484 \\
0.0028 & 0.0000 & 0.0285 & 0.4074 & 0.5613
\end{array}\right]
$$

533 Similar to the $F B_{l}$ calculation, we get:

534

$$
F B=\left[\begin{array}{llllll}
0.0000 & 0.0000 & 0.0446 & 0.4170 & 0.5385 \\
0.0018 & 0.0009 & 0.0915 & 0.4241 & 0.4818 \\
0.0005 & 0.0040 & 0.0682 & 0.4018 & 0.5254 \\
0.0017 & 0.0013 & 0.0310 & 0.4617 & 0.5041 \\
0.0014 & 0.0005 & 0.0995 & 0.4312 & 0.4674 \\
0.0014 & 0.0000 & 0.0509 & 0.4486 & 0.4991
\end{array}\right]
$$

By using Equations (26), the factors weights value, the result set of second-layer comprehensive evaluation is as follows

$$
B_{2}=W \cdot F B=
$$

$(0.1764,0.1714,0.1685,0.1627,0.1651,0.1559) \cdot$

$\left[\begin{array}{llllll}0.0000 & 0.0000 & 0.0446 & 0.4170 & 0.5385 \\ 0.0018 & 0.0009 & 0.0915 & 0.4241 & 0.4818 \\ 0.0005 & 0.0040 & 0.0682 & 0.4018 & 0.5254 \\ 0.0017 & 0.0013 & 0.0310 & 0.4617 & 0.5041 \\ 0.0014 & 0.0005 & 0.0995 & 0.4312 & 0.4674 \\ 0.0014 & 0.0000 & 0.0509 & 0.4486 & 0.4991\end{array}\right]$

$$
=(0.0011,0.0011,0.0644,0.4302,0.5030)
$$

538 Where, $B_{2}$ represent the fuzzy comprehensive results of the second evaluation. 


\section{Discussion}

\section{$540 \quad 5.1 \quad$ Comparative analysis of FCE results following BBS intervention}

The result of second evaluation reveals that the probability of the sustainable safety performance very high is 0.5030 ; the probability of 'high', 'medium', 'low' and 'very low' are $0.4302,0.0644,0.0011$ and 0.0011 . The result showing that the sustainable safety performance is assessed as very high. The twice assessment results are shown in Fig. 7.

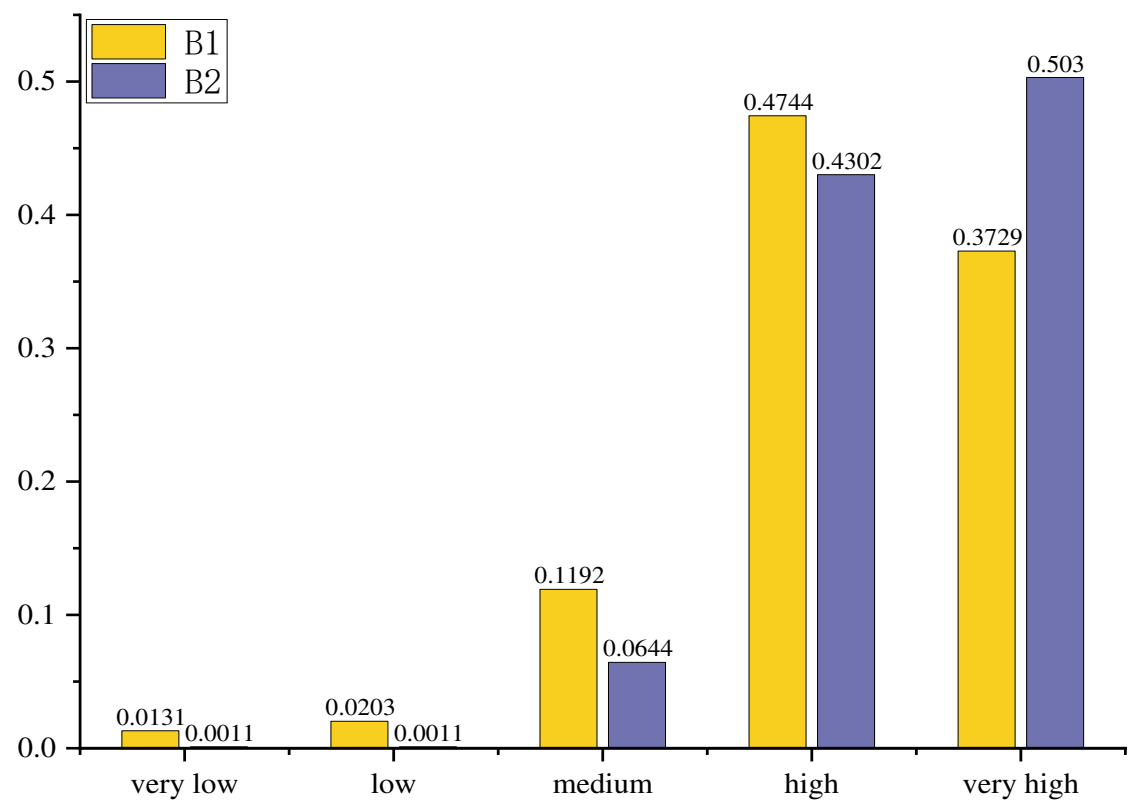

Fig. 7. Histogram of the sustainable safety performance fuzzy evaluation.

Fig. 7 illustrate that the sustainable safety performance of the second assessment is better than that of the first. During the implementation of BBS, there are no examples of rejecting safety interventions. This intervention has a significant influence on the management systems, raising safety climate and increasing safety communication. The results show that BBS is a sustainable safety management. In this intervention process, it is not necessary to stop working, but to take measures to correct employees' unsafe acts. The fact that unsafe acts can be regarded as a social and psychological phenomenon. Especially in recent years, evaluation methods in group psychology and cognitive psychology have become more and more popular (Calvo garz ó n et al., 2008). Previous studies have shown that it is feasible to combine management, mathematics and psychology, and that interdisciplinary insights can be obtained. The advantage of fuzzy mathematics is that it can integrate human thinking and objective data into the process 
560 of cognitive psychology. This integration can more richly describe human factors, sus-

561 tainable safety development and BBS management. Previous safety management stud562 ies usually emphasized external factors and rarely considered the possibility of cogni563 tive psychological effects.

564 In addition to the external factors, BBS intervention is mainly affected by internal 565 psychological factors, such as value congruence, psychological identity and cognitive 566 process. Psychological identity is closely related to safety climate, safety communica567 tion and safety culture. This means that BBS intervention is more likely to succeed in 568 a plant with a positive safety climate. Value consistency is likely to lead to satisfaction 569 and recognition, so as to improve sustainable safety performance. However, the worksistent in the implementation of the BBS intervention. Therefore, employees may be too hasty to perfunctory BBS intervention. On the other hand, in the process of BBS intervention, safety ability is a key regulatory factor between safety behavior and sustainable safety performance. This means that unsafe act is not only a problem of psychological motivation, but may also be a problem of ability. There are some activitybased training programs to help employees improve their safety ability. As mentioned above, BBS intervention and psychological identity are two complementary methods to improve unsafe act. Many factors in the evaluation model (Figure 12) are related to the dimension of psychological identity. Therefore, psychological identity also is the supplement of BBS intervention, and explains the mixed effect from the perspective of psychology.

With the improvement of psychological identity, employees experience fewer conflicts and are more willing to cooperate with the BBS intervention. Thus, managers are motivated to pursue higher safety performance because it is in line with their psychological identity and personal interests. Employees are more driven by internal psychological identity than external punishment or reward. Psychological identity is relatively stable. Therefore, when the BBS program is implemented in plant with consistent psychological identity, its safety performance is more likely to be sustainable. In addition, the consistency of psychological identity can also feedback positive group norms. Positive group norms can reduce work conflicts and violations because all employees expect to work continuously and safely under occupational pressure (Goh et al. 2015). To sum up, psychological identity, willingness to pursue sustainable safety performance, positive group norms and human factors are crucial to the success of BBS intervention.

\subsection{Weight analysis}

Fig 8 and 9 show the local and global weights of the research factors and sub-factors obtained by applying the FAHP techniques. Obviously, each factor with higher weight value is more important the decision-making process. The global weights of all subfactor are the results by multiplying the local weights of all sub-factor with the weights of the factor to which it belongs. 


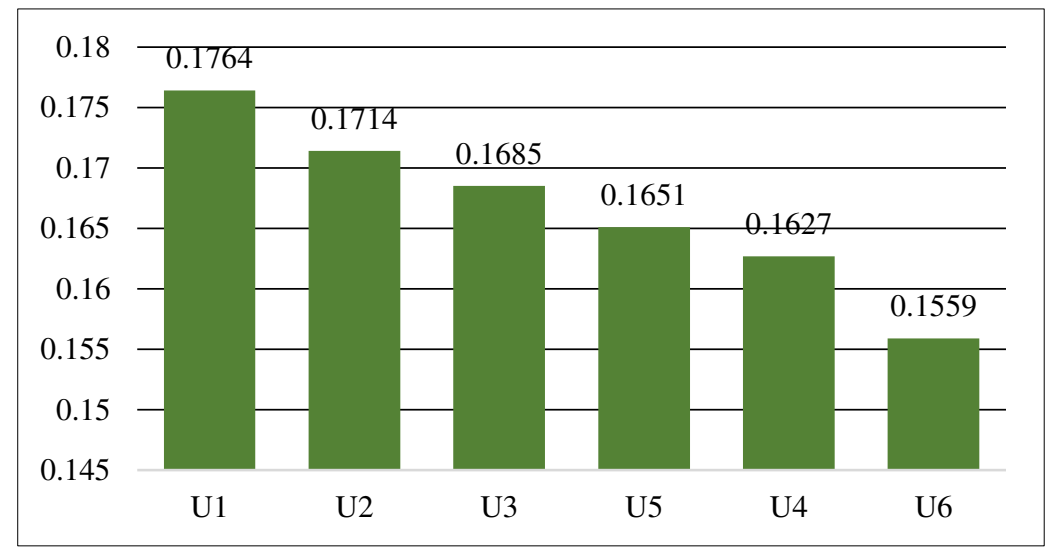

Fig. 8. Local weights of the factors.

In Fig. 8, we rank the local weights of all factors from large to small. We realize that $U_{1}$ (employees' psychological behavior) is the highest percent of effect on the sustainable safety performance rank among all factors ( $U_{1}$ ranks highest in the local weight), followed by $U_{2}$ (Process safety education), $U_{3}$ (Working atmosphere), $U_{5}$ (Equipment factors), $U_{4}$ (Management factors) and $U_{6}$ (Employee's comprehensive quality). Thus, $U_{1}$ (Employees' psychological behavior) and $U_{2}$ (Process safety education) are the key behavior factors must to be considered for the employees. This means that the safety managers should mainly pay attention to the psychological and educational issues. Psychological pressure may have negative effects on an individual physical and mental health, such as increased distractibility, lower concentration, and more prone to burnout. When employees experience general psychological pressure, they are unlikely to initiate accident reporting or safely use equipment. If employees are face with greater psychological pressure, the plant will face more lost working days, absenteeism and lower safety performance.

In addition, $U_{2}$ (Process safety education) is also considered to be one of the most important factors for the practice of sustainable safety development in the chemical industry. Process safety education' is generally defined as learning safety principles and operating disciplines to prevent major accidents and casualties in the process industry (Mkpat et al. 2018). Process safety education aims to improve the understanding of process safety principles, promote safety knowledge sharing and improve technical level (Nesheim and Gressgård, 2014). In addition to improving safety performance, process safety education indirectly promotes many areas of safety culture. It sustains industry reliability, improves productivity, and enhances the sustainable safety development of the chemical industry. Consequently, these plants effectively convey safety information through meetings and training, quickly solve safety problems, and regard safety training as an investment. It is also observed that safety climate significantly affected sustainable safety performance. The traditional safety climate only focuses on the physical aspects of safety, and now it has been extended to the psychological safety atmosphere, which focuses on the psychological aspects of health and safety (Yaris et al. 2020). A positive safety climate gives priority to psychological safety, encourages 
633

psychological-oriented safety behavior, and pays attention to sustainable safety performance. Hence, these three factors are considered to be the key to achieving sustainable safety development.

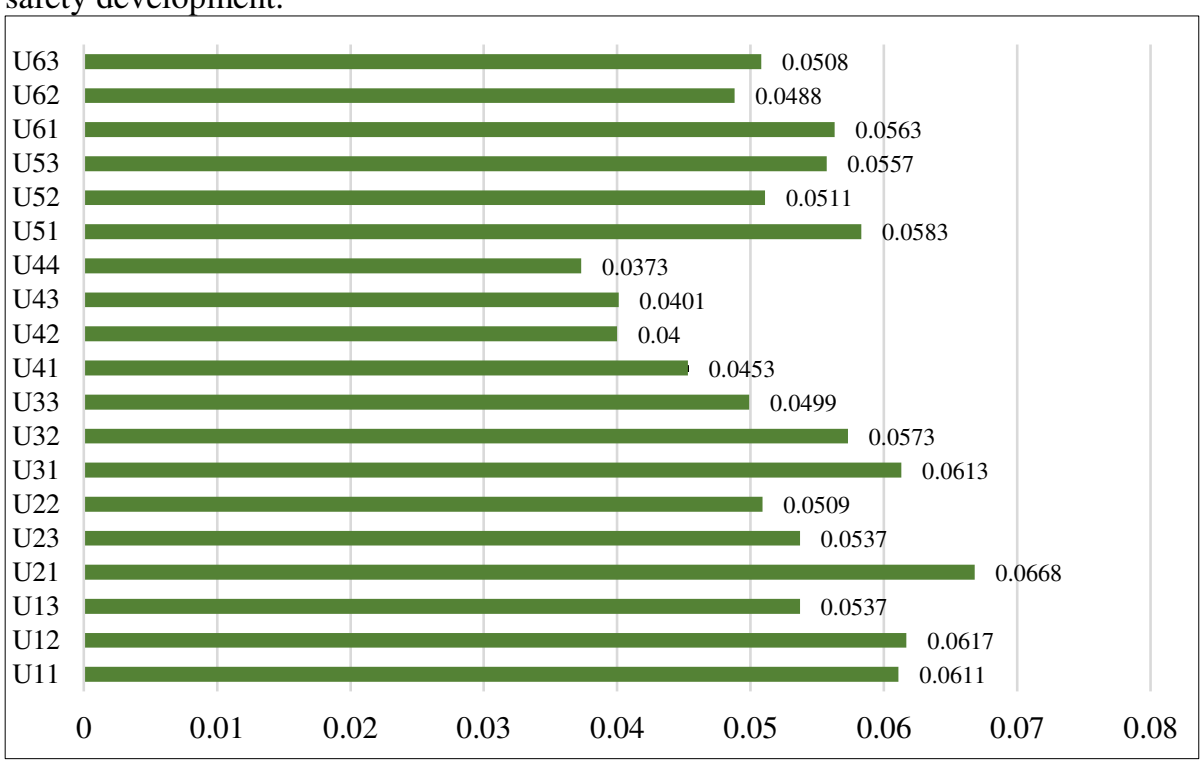

Fig. 9. Global weights of factors and sub-factors.

Fig. 9, $U_{21}$ (Frequency and intensity of safety training) are considered to be the most influential factors in the global weight part, which is followed by $U_{12}$ (Personal psychological quality), $U_{31}$ (Performance of workers in daily safety behavior) and $U_{11}$ (Personal attention at work). Conversely, $U_{44}$ (Corporate reward and punishment of safety production), $U_{42}$ (Improvement degree of safety regulations), $U_{43}$ (Corporate supervision of safety production) and $U_{41}$ (Improvement degree of safety production plans) have less influence. $U_{21}$ (Safety training) is actually formed and developed for major accidents in the chemical process industry. Accidents in this dangerous industry can have serious consequences for the personnel, surrounding environment, and plant assets. In terms of preventing major incidents, safety training has developed various tools, approaches and procedures aimed at removing human errors and technical design defect, as well as safety management systems. The dangers of the chemical industry led to complexity tasks, including the rate of information change, information diversity, increase of information volume. These factors demand the employees to make greater efforts lead to greater stress, and may lead to burnout, unsafe acts, fatigue and incidents. For $U_{12}$ (Personal psychological quality) and $U_{31}$ (Performance of workers in daily safety behavior), high psychological quality and safety behavior can hold a positive attitude towards stress, and employees will not experience any negative results.

Furthermore, according to the idea of self-regulation theory, for $U_{11}$ (Personal attention at work), attention mechanism and behavior process can guide individuals to develop towards the safe direction of goal setting. For $U_{44}$, Komaki et al. (1978) proposed a method of rewarding employees to reduce unsafe acts in the industry. Rewards and 
660 punishments create a psychological environment that encourages employees to modify

661 their target behavior. However, this theory does not explain why a rewards or punish662 ment modify a behavior and what makes it work. For $U_{42}$ (Improvement degree of 663 safety regulations), $U_{43}$ (Corporate supervision of safety production) and $U_{41}$ (Improve664 ment degree of safety production plans). The $U_{41}$ has a lowest weight value of 0.12 , 665 while the $U_{42}$ and $U_{43}$ have weights of 0.15 and 0.5 . This means that decision makers 666 take into account the cognition and psychology of employees, and the factors related to 667 safety production have little effect on behavior improvement.

\subsection{Analysis of the recordable incidents in UK}

The incidence of manufacturing related accidents and injuries also remains high. Therefore, in any realistic prospect of safety intervention in this complex industry, the understanding of the root cause of the accident is still indispensable. Pickup et al. (2020) uses the personal diary method to record and analyze real-time data of safety incidents (Pickup et al. 2020). This study qualitatively explores the safety related events recorded by employees for the first time, so as to identify the perceived potential risk and find human errors. Taking a car manufacturing site in the UK as an example, Pickup et al recorded and analyzed 176 incidents and classified them according to the event type in Table 13.

Table 13 Percentage and frequency of incident types in log data (Pickup et al. 2020).

\begin{tabular}{llll}
\hline & Frequency & $\%$ & Official Data \\
\hline Unsafe Condition & 50 & 28.41 & - \\
Unsafe Act & 67 & 38.07 & - \\
Near Miss & 46 & 26.14 & 12 \\
Accident & 13 & 7.39 & 3 \\
Personally experienced & 42 & 23.86 & - \\
Total & 176 & 100.00 & 15 \\
\hline
\end{tabular}

The most common type of incident is unsafe acts, accounting for $38.07 \%$, followed by unsafe conditions $(28.41 \%)$. The proportion of near misses $(\mathrm{N}=46)$ is high compared with accidents $(\mathrm{N}=13)$, accounting for $26 \%$ of the data, and exceeds the official data reported in the same time period ( $\mathrm{N}=12$ and $\mathrm{N}=3$, respectively). Therefore, exploring the causes of accidents is still an indispensable part of injury reduction and safety improvement, especially within complex manufacturing and chemical industries. This study reveals that a more comprehensive taxonomy and model need to be integrated into accident investigation and analysis, so as to explore the diversity of human error and cognitive performance at the personal level. Meanwhile, human factors describe the correlation between safety climate and unsafe act, including safety culture and manager quality. Consequently, the study makes BBS intervention possible in daily unsafe acts, thus clarifying a new view of human factors at the system and individual levels.

\subsection{Analysis of the recordable accident data in France}


693 Accidents can cause disastrous damage to environment, human health and economy in

the chemical industry. In order to prevent such incidents in the industry, it is necessary to review and analyze the past accident data. ARIA (Analysis, Research and Information on Accidents) database is considered to be one of the important technical accident databases in Europe. ARIA is a huge database managed and maintained by the French Ministry of ecology, sustainable development and energy. The database records more than 43000 accidents occurred in the world and in France. Dakkoune et al. (2018) collected and selected 169 safety related events in the French database ARIA. These safety related events occurred between 1974 and 2014. Dakkoune et al. (2018) analyzed the causes and consequences of these events. According to the type of event and the cause of the event, the risk distribution is shown in Figure 10.

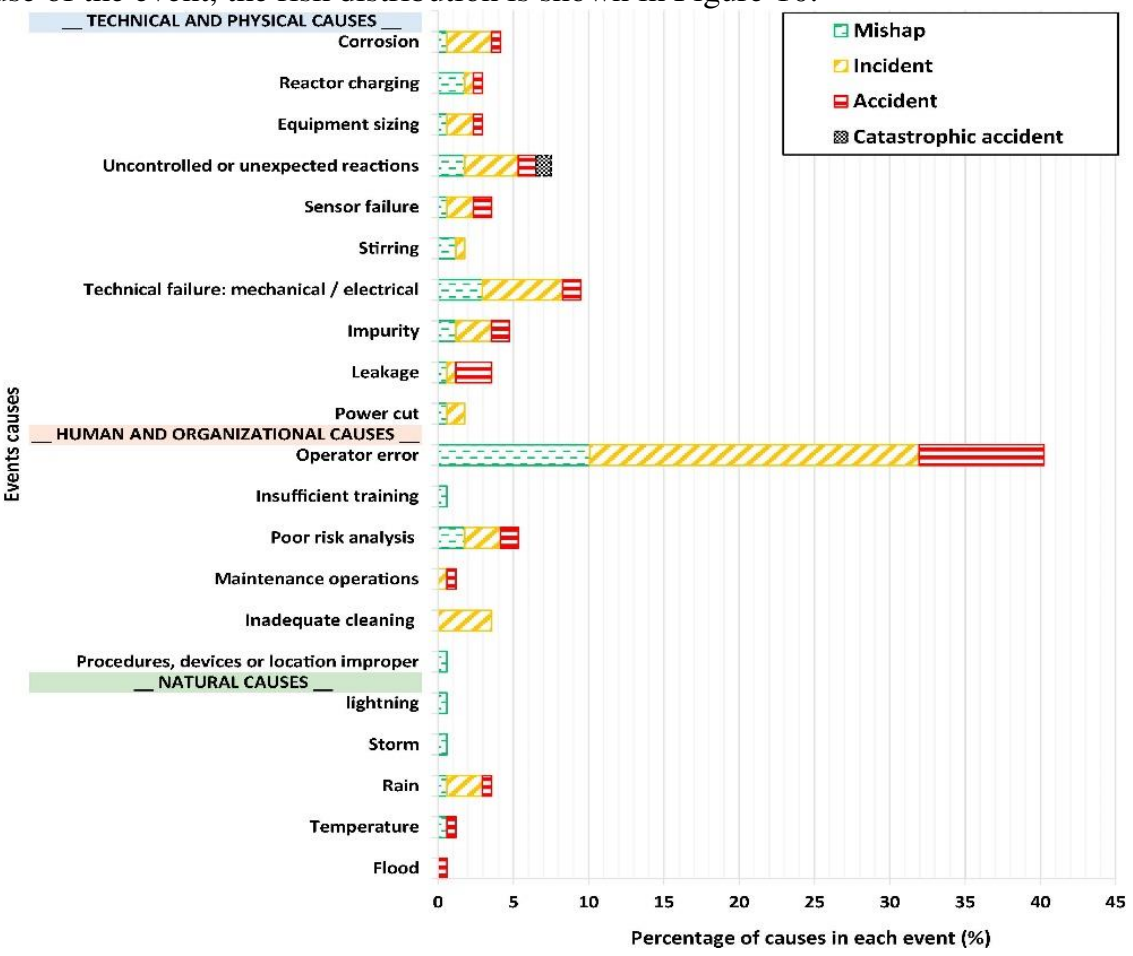

Fig. 10. Distribution of initial causes for each event (Dakkoune et al. 2018).

Obviously, the main initial cause of events in the chemical industry sector is operator error (about $40 \%$ of events). The other initial causes contain technical and physical causes, as well as human and organizational causes, which are respectively classified as the following uncontrolled or unexpected reactions, technical failure, insufficient risk analysis and corrosion, etc. In natural causes, most risks are less than $4 \%$, although natural phenomena are often difficult to predict and generally destructive.

The chemical industry needs to pay more attention to and prevent these risks related to human factors. Because human behavior is complex and uncontrollable, it interacts with other external factors, such as equipment, colleagues, management, environment, 
etc. In order to reduce accidents and injuries related to human factors, the research of approaches to improve the unsafe act of employees is crucial. In fact, it is difficult to design and apply digital systems to eliminate all human errors in daily work. On the other hand, a BBS intervention process needs to be proposed to identify and improve unsafe behaviors. For the government, this BBS approach can also be used as a starting point for developing safety management strategy, in order to prevent and reduce the number of safety related events in the chemical industry.

\section{Conclusions}

This paper proposes a comprehensive sustainable safety performance assessment framework based on BBS management, FAHP and FCE, which included 6 factors and 19 sub-factors. The evaluation results further show the importance of BBS management to the implementation and achievement of sustainable safety development in the petrochemical plant. The results of this study can be concluded as follows:

(1) Due to its unique complexity and danger, petrochemical industry is the most prone to major accidents in the world, so it is urgent to improve the performance of employees' safety behavior. However, the traditional BBS research mainly focuses on the behavior analysis after the occurrence of an accidents, because there is no tool to collect employees' behavior data from on site. In addition, the essence of employees' safety behavior evaluation involves many processes in the petrochemical plant. Therefore, we proposed the model overcome this limitation and it can record, monitor and assess employees' safety behaviors. In practical application, with the collection of behavioral data, the model is used to quantitatively evaluate the sustainable safety performance of employees.

(2) The model systematically combines the knowledge and experience of the expert team to calculate the weight of safety behavior factors. Weight ranking can also provide safety strategy to managers in a reasonable, scientific and effective manner. In addition, in order to reduce industry accidents, the petrochemical plant is becoming more and more employee-centered and strive to improve their sustainable safety performance. In conclusion, the model determines the sustainable safety performance of employees based on calculated weight and obtained data, and its data sources are both objective and subjective. These evaluation results can help employees realize that safety is everyone's responsibility and safety is above all else.

(3) To prevent potential risky behaviors of employees, this paper applies a more professional and systematic approach to assess sustainable safety performance of employees. BBS management will ensure that employees have adequate control and improve of safety behavior and psychological health. The BBS process emphasizes the operations management, planned maintenance, correct use of equipment and risk analysis. This requires develop guidelines for safety behavior, creation of unified teams with opened safety communication and shared responsibility for employee safety, and leaders and managers to consider the BBS program regard as a benefit rather than a burden. 


\section{$7 \quad$ Limitations of this study}

757 Due to state-owned company and policy constraints, the intervene time is only 14 days.

758 The assessment data and frequency of employee behaviors is limited. As a result, the 759 proposed BBS management lacks some sustainability in collect employee's behavior 760 data. Moreover, the case study was implemented in state-owned company and mostly 761 involved dangerous petrochemical experiments at the time of the study. In some ways, 762 the BBS process may be not allowed and interrupted. Finally, the company culture and 763 the nature of the company may influence the sustainability and effectiveness of the 764 employees-focused BBS management and such influence needs further research.

Author contribution All authors have contributed to the study. Junqiao Zhang is the main contributor to writing the manuscript. Xuebo Chen checked the grammar and language of this manuscript and collected data in the Petrochemical plant. Qiang Qu analyzed and computed the data.

Funding This study was supported by the National Science Fund of China $(71571091,71771112)$

Availability of data and material All data generated or analyzed during this study are included in this published article. More detail data may be provided by the author upon reasonable request and permission

\section{Compliance with ethical standards}

Ethics approval Not applicable

Consent for publication Not applicable

Consent for participate Not applicable

Competing interests The authors declare no competing interests

\section{$8 \quad$ References}

Alkaissy M, Arashpour M, Ashuri B, Bai Y, Hosseini R (2020) Safety management in construc tion: 20 years of risk modeling. Safety science 129:104805

Batoo KM, Pandiaraj S, Muthuramamoorthy M, Raslan EH, Krishnamoorthy S (2021) Behaviorbased swarm model using fuzzy controller for route planning and E-waste collection. Environmental Science and Pollution Research 1-15

Baysari MT, McIntosh AS, Wilson JR (2008) Understanding the human factors contribution to railway accidents and incidents in Australia. Accident Analysis \& Prevention 40(5):17501757

Brandhorst S, Kluge A (2021) When the tension is rising: a simulation-based study on the effects of safety incentive programs and behavior-based safety management. Safety 7(1):9 
Brosschot JF, Verkuil B, Thayer JF (2018) Generalized unsafety theory of stress: Unsafe envi ronments and conditions, and the default stress response. International journal of environmental research and public health 15(3):464

Chang CW, Wu CR, Lin HL (2009) Applying fuzzy hierarchy multiple attributes to construct an expert decision-making process. Expert Systems with Applications 36(4):7363-7368

Chang DY (1992) Extent analysis and synthetic decision. Optimization techniques and applications 1(1):352-355

Chang DY (1996) Applications of the extent analysis method on fuzzy AHP. European journal of operational research 95(3):649-655

Chen D, Tian H (2012) Behavior based safety for accidents prevention and positive study in China construction project. Procedia Engineering 43:528-534

Chen JF, Hsieh HN, Do QH (2015) Evaluating teaching performance based on fuzzy AHP and comprehensive evaluation approach. Applied Soft Computing 28:100-108

Chen T, Wu HC (2021) Fuzzy collaborative intelligence fuzzy analytic hierarchy process approach for selecting suitable three-dimensional printers. Soft Computing 25(5):4121-4134

Chien F, Ngo QT, Hsu CC, Chau KY, Iram R (2021) Assessing the mechanism of barriers towards green finance and public spending in small and medium enterprises from developed countries. Environmental Science and Pollution Research 1-16

Choudhry RM (2014) Behavior-based safety on construction sites: A case study. Accident analysis \& prevention 70:14-23

Chua DK, Goh YM (2004) Incident causation model for improving feedback of safety knowledge. Journal of construction engineering and management 130(4):542-551

Dağdeviren M, Yüksel İ (2008) Developing a fuzzy analytic hierarchy process (AHP) model for behavior-based safety management. Information sciences 178(6):1717-1733

Dakkoune A, Vernières-Hassimi L, Leveneur S, Lefebvre D, Estel L (2018). Risk analysis of French chemical industry. Safety science 105:77-85

Dasgupta P, Muhammad J, Mauerman LA (2020) Utilizing Skill To Improve Behavior-Based Safety. Professional Safety 65(06):48-52

Dodoo JE, Al-Samarraie H (2021) A systematic review of factors leading to occupational injuries and fatalities. Journal of Public Health 1-15

Favero N, Meier KJ, O'Toole JrLJ (2016) Goals, trust, participation, and feedback: Linking internal management with performance outcomes. Journal of Public Administration Research and Theory 26(2):327-343

Feng Z, Ji N, Luo Y, Sze NN, Tian J, Zhao C (2020) Exploring the influencing factors of public traffic safety awareness in China. Cognition, Technology \& Work 1-12

Garlapati A, Siddiqui N, Al-Shatti F (2013) Behavioral study of diverse workforce towards various health, safety and environment engagement strategies in upstream oil and gas industries. International Journal of Scientific and Engineering Research 4(7):1768-1779

Geller ES (1999) Behavior-based safety: confusion, controversy, and clarification. Occupational Health \& Safety 68(1):40-49

Geller ES (2002) Behavior-based safety in industry: realizing the large-scale potential of psychology to promote human welfare. Applied \& Preventive Psychology 10(2):87-105

Geller ES (2005). Behavior-based safety and occupational risk management. Behavior modification 29(3):539-561

Goh YM, Tan S, Lai KC (2015) Learning from the Bhopal disaster to improve process safety management in Singapore. Process Safety and Environmental Protection 97:102-108

Goyal S, Garg D, Luthra S (2021) Sustainable production and consumption: Analysing barriers and solutions for maintaining green tomorrow by using fuzzy-AHP-fuzzy-TOPSIS hybrid framework. Environment, Development and Sustainability 1-47 
Gul M, Guven B, Guneri AF (2018) A new Fine-Kinney-based risk assessment framework using FAHP-FVIKOR incorporation. Journal of Loss Prevention in the Process Industries 53:316

Hagge M, McGee H, Matthews G, Aberle S (2017). Behavior-based safety in a coal mine: The relationship between observations, participation, and injuries over a 14-year period. Journal of Organizational Behavior Management 37(1):107-118

Harsini AZ, Ghofranipour F, Sanaeinasab H, Shokravi FA, Bohle P, Matthews LR (2020) Factors associated with unsafe work behaviours in an Iranian petrochemical company: perspectives of workers, supervisors, and safety managers. BMC Public Health 20(1):1-13

Heinrich HW. Industrial accident prevention: A scientific approach. New York, NY: McGrawHill (1931)

Hsu TH, Chen CH, Liao WC (2021). A Fuzzy MCDM Analytic Model for Building Customers' Brand Attachment Preference in Car Firms. International Journal of Fuzzy Systems 1-13

Hsu WKK, Huang SHS, Tseng WJ (2016) Evaluating the risk of operational safety for dangerous goods in airfreights-A revised risk matrix based on fuzzy AHP. Transportation research part D: transport and environment 48:235-247

Hu Y, Wu L, Pan X, Wang Z, Xu X (2021) Comprehensive Evaluation of Cloud Manufacturing Service Based on Fuzzy Theory. International Journal of Fuzzy Systems:1-10

Iqbal MI, Isaac O, Al Rajawy I, Khuthbuddin S, Ameen A (2021) Hazard identification and risk assessment with controls (Hirac) in oil industry-A proposed approach. Materials Today: Proceedings 44:4898-4902

Kahraman C, Ertay T, Büyüközkan G (2006) A fuzzy optimization model for QFD planning process using analytic network approach. European Journal of Operational Research 171(2):390-411

Karthick M, Robert TP, Kumar S (2020) HFACS-based FAHP implementation to identify critical factors influencing human error occurrence in nuclear plant control room. Soft Computing 24(21):16577-16591

Kauffman A, Gupta MM (1991) Introduction to fuzzy arithmetic: theory and application. NY: Van Nostrand Reinhold Company, New York (1991)

Kelly D, Efthymiou M (2019) An analysis of human factors in fifty controlled flight into terrain aviation accidents from 2007 to 2017. Journal of safety research 69:155-165

Khashei-Siuki A, Sharifan H (2020) Comparison of AHP and FAHP methods in determining suitable areas for drinking water harvesting in Birjand aquifer. Iran. Groundwater for Sustainable Development 10:100328

Komaki J, Barwick KD, Scott LR (1978) A behavioral approach to occupational safety: pinpointing and reinforcing safe performance in a food manufacturing plant. Journal of applied Psychology 63(4):434

Lee P C, Wei J, Ting HI, Lo TP, Long D, Chang LM (2019) Dynamic analysis of construction safety risk and visual tracking of key factors based on behavior-based safety and building information modeling. KSCE Journal of Civil Engineering 23(10):4155-4167

Li S, Wu X, Wang X, Hu S (2020) Relationship between social capital, safety competency, and safety behaviors of construction workers. Journal of construction engineering and management 146(6):04020059.

Lyu HM, Shen SL, Zhou A, Yang J (2020). Risk assessment of mega-city infrastructures related to land subsidence using improved trapezoidal FAHP. Science of The Total Environment 717:135310

Ma Z, Zhu J, Zhang S (2021) Probabilistic-based expressions in behavioral multi-attribute decision making considering pre-evaluation. Fuzzy Optimization and Decision Making 20(1):145-173 
McSween T, Moran DJ (2017) Assessing and preventing serious incidents with behavioral science: enhancing Heinrich's triangle for the 21 st century. Journal of Organizational Behavior Management 37(3-4):283-300

Mirzaei Aliabadi M, Aghaei H, Kalatpour O, Soltanian AR, Nikravesh A (2020) Analysis of human and organizational factors that influence mining accidents based on Bayesian network. International journal of occupational safety and ergonomics 26(4):670-677

Mkpat E, Reniers, Cozzani V (2018) Process safety education: A literature review. Journal of Loss Prevention in the Process Industries 54:18-27

Mohsenzadeh A, Motameni H, Er MJ (2019) Retraction Note to: A New Trust Evaluation Algorithm Between Cloud Entities Based on Fuzzy Mathematics. International Journal of Fuzzy Systems 6(21):1988-1988

Navidian A, Rostami Z, Rozbehani N (2015) Effect of motivational group interviewing-based safety education on Workers' safety behaviors in glass manufacturing. BMC public health 15(1): $1-7$

Nesheim T, Gressgård LJ (2014) Knowledge sharing in a complex organization: Antecedents and safety effects. Safety science 62:28-36

Nguyen HT, Md Dawal SZ, Nukman Y, Aoyama H, Case K (2015) An integrated approach of fuzzy linguistic preference based AHP and fuzzy COPRAS for machine tool evaluation. PloS one 10(9):e0133599

Norton TA, Zacher H, Parker S.L, Ashkanasy NM (2017) Bridging the gap between green behavioral intentions and employee green behavior: The role of green psychological climate. Journal of Organizational Behavior 38(7):996-1015

Pickup S, Paton K, Hayes C, Morrison B (2020) A day in the life of frontline manufacturing personnel: A diary-based safety study. Safety Science 132:104992

Pramanik D, Mondal SC, Haldar A (2020) A framework for managing uncertainty in information system project selection: An intelligent fuzzy approach. International Journal of Management Science and Engineering Management 15(1):70-78

Reason J (2000) Human error: models and management. Bmj 320(7237):768-770

Reason J, Hollnagel E, Paries J (2006) Revisiting the Swiss cheese model of accidents. Journal of Clinical Engineering 27(4):110-115

Rodriguez MA, Bell J, Brown M, Carter D (2017) Integrating behavioral science with human factors to address process safety. Journal of Organizational Behavior Management. 37(34):301-315

Rollinson AN (2018) Fire, explosion and chemical toxicity hazards of gasification energy from waste. Journal of Loss Prevention in the Process Industries 54:273-280

Saaty TL. The Analytic Hierarchy Process. McGraw-Hill Companies Inc, New York (1980)

Santoyo-Castelazo E, Azapagic A (2014) Sustainability assessment of energy systems: integrating environmental, economic and social aspects. Journal of Cleaner Production 80:119-138

Sauer J, Chavaillaz A, Wastell D (2016) Experience of automation failures in training: effects on trust, automation bias, complacency and performance. Ergonomics 59(6):767-780

Scianca N, Ferrari P, De Simone D, Lanari L, Oriolo G (2021) A behavior-based framework for safe deployment of humanoid robots. Autonomous Robots 1-22

Sharma A, Sharma P, Sharma A, Tyagi R, Dixit A (2017) Hazardous Effects of Petrochemical Industries: A. petrochemical technology 13(3):1-7

Shaygan A, Testik ÖM (2019) A fuzzy AHP-based methodology for project prioritization and selection. Soft computing 23(4):1309-1319

Shete PC, Ansari ZN, Kant R (2020) A Pythagorean fuzzy AHP approach and its application to evaluate the enablers of sustainable supply chain innovation. Sustainable Production and Consumption 23:77-93 
935 Van Laarhoven PJ, Pedrycz W (1983) A fuzzy extension of Saaty's priority theory. Fuzzy sets and Systems 11(1-3):229-241

Wang B, Li D, Wu C (2020) Characteristics of hazardous chemical accidents during hot season in China from 1989 to 2019: A statistical investigation. Safety science 129:104788

Wang B, Song J, Ren J, Li K, Duan H (2019) Selecting sustainable energy conversion technologies for agricultural residues: A fuzzy AHP-VIKOR based prioritization from life cycle perspective. Resources, Conservation and Recycling 142:78-87

Wang B, Wu C, Shi B, Huang L (2017) Evidence-based safety (EBS) management: A new approach to teaching the practice of safety management (SM). Journal of safety research 63:21-28

Wang X, Xing Y, Luo L, Yu R (2018) Evaluating the effectiveness of Behavior-Based Safety education methods for commercial vehicle drivers. Accident Analysis \& Prevention 117:114-120

Yaris C, Ditchburn G, Curtis GJ, Brook L (2020) Combining physical and psychosocial safety: A comprehensive workplace safety model. Safety Science 132:104949

Yu M, Li JZ (2019) Influence of behavior-based safety management on improving of environmentally coal miner's mental health. Ekoloji 28(107):4513-4520

Zadeh LA (1965) FUZZY SETS. Information \& Control 8(3):338-353

Zadeh LA (1978) Fuzzy sets as a basis for a theory of possibility. Fuzzy sets and systems 1(1):328

Zhang P, Li N, Fang D, Wu H (2017) Supervisor-focused behavior-based safety method for the construction industry: Case study in Hong Kong. Journal of Construction Engineering and Management 143(7):05017009 